\title{
Role of endoplasmic reticulum stress in disuse osteoporosis
}

Jie Li ${ }^{a, b}$, Shuang Yanga,b, Xinle Li ${ }^{a, b, c}$, Daquan Liu ${ }^{a, b, d}$, Zhe Gao ${ }^{a}$, Xiaoyu Zhao $^{a}$, Jiuguo Zhang ${ }^{a}$, Fanglin Gou ${ }^{a}$, Hiroki Yokota ${ }^{\mathrm{e}}$, and Ping Zhanga,b,c,e*

aDepartment of Anatomy and Histology, School of Basic Medical Sciences, Tianjin Medical University, Tianjin 300070, China

bTEDA International Cardiovascular Hospital, Chinese Academy of Medical Sciences \& Peking Union Medical College, Tianjin 300457, China

cKey Laboratory of Hormones and Development (Ministry of Health), Tianjin Key Laboratory of Metabolic Diseases, Tianjin Medical University, Tianjin 300070, China

${ }^{\mathrm{d}}$ Department of Pharmacology, Institute of Acute Abdominal Diseases, Tianjin Nankai Hospital, Tianjin 300100, China

eDepartment of Biomedical Engineering, Indiana University-Purdue University Indianapolis, IN 46202, USA

KEY WARDS: Endoplasmic reticulum stress; Eukaryotic translation initiation factor 2 $\alpha$; Osteoporosis; Disuse; Hindlimb unloading; Salubrinal

Running title: Endoplasmic Reticulum Stress in Osteoporosis

*Corresponding Author: Ping Zhang, MD

Department of Anatomy and Histology

School of Basic Medical Sciences

Tianjin Medical University

22 Qixiangtai Road

Tianjin 300070, China

Phone: 86-22-83336818

Fax: 86-22-83336810

E-mail: pizhang2008@163.com

Abbreviations: eIF2a, Eukaryotic translation initiation factor 2 alpha; ER, endoplasmic reticulum; CHOP, C/EBP homologous protein; RANKL, Receptor

This is the author's manuscript of the article published in final edited form as:

Li, J., Yang, S., Li, X., Liu, D., Wang, Z., Guo, J., ... \& Gou, F. (2017). Role of endoplasmic reticulum stress in disuse osteoporosis. Bone, 97, 2-14. https://doi.org/10.1016/j.bone.2016.12.009 
activator of nuclear factor kappa-B ligand; M-CSF, Macrophage-colony stimulating factor; TRAP, Tartrate resistant acid phosphatase; ALP, Alkaline phosphatase; ATF4, Activating transcription factor 4 


\section{ABSTRACT}

Osteoporosis is a major skeletal disease with low bone mineral density, which leads to an increased risk of bone fracture. Salubrinal is a synthetic chemical that inhibits dephosphorylation of eukaryotic translation initiation factor 2 alpha (eIF2 $\alpha$ ) in response to endoplasmic reticulum (ER) stress. To understand possible linkage of osteoporosis to ER stress, we employed an unloading mouse model and examined the effects of salubrinal in the pathogenesis of disuse osteoporosis. The results presented several lines of evidence that osteoclastogenesis in the development of osteoporosis was associated with ER stress, and salubrinal suppressed unloading-induced bone loss. Compared to the age-matched control, unloaded mice reduced the trabecular bone area/total area (B.Ar/T.Ar) as well as the number of osteoblasts, and they increased the osteoclasts number on the trabecular bone surface in a time-dependent way. Furthermore, a significant correlation of ER stress with the differentiation of osteoblasts and osteoclasts was observed. Administration of salubrinal suppressed the unloading-induced decrease in bone mineral density, B.Ar/T.Ar, and mature osteoclast formation. Salubrinal also increased the colony-forming unit-fibroblasts and osteoblasts. It reduced the formation of mature osteoclasts, suppressed their migration and adhesion, and increased phosphorylation of eIF $2 \alpha$. While unloading-induced ER stress reduced the number of osteoblasts and increased the number of osteoclasts, salubrinal suppressed those changes. A TUNEL assay together with CHOP expression indicated that ER stress-induced osteoblast apoptosis was rescued by salubrinal. Collectively, the results support the notion that ER stress plays a key role in the pathogenesis of disuse osteoporosis, and salubrinal attenuates unloading-induced bone loss by altering proliferation and differentiation of osteoblasts and osteoclasts via eIF2 $\alpha$ signaling. 


\section{Introduction}

Stress to the endoplasmic reticulum (ER) is recognized as cellular insult to a protein-folding factory, responsible for the biosynthesis, folding, assembly and modification of numerous soluble proteins and membrane proteins [1]. Disturbance to normal functions of the ER leads to an evolutionarily conserved stress response, the unfolded protein response, which is primarily aimed at damage compensating but may eventually trigger cell death if the dysfunction is severe or prolonged[2, 3].Although the ER stress has been reported to be linked to various diseases such as diabetes[4,5], neurodegenerative diseases[6], and osteogenesis imperfecta [7], the role of the ER stress in the pathogenesis of osteoporosis still remains unclear.

Osteoporosis is characterized by reduced bone mass, alterations in the microarchitecture of bone tissue, reduced bone strength, and an increased risk of fracture $[8,9]$. Bone remodeling is a continuous bone resorbing and rebuilding process, undertaken mainly by bone-resorbing osteoclasts and bone-forming osteoblasts [10]. Diminished bone formation and/or excessive bone resorptionduring bone remodeling results in osteoporosis. Some murine models for bone diseases exhibit useful insights into possible linkage of osteoporotic pathogenesis to the ER stress in osteoblasts [11]. For instance, increased apoptotic death of osteoblasts is observed in postmenopausal osteoporosis as well as glucocorticoid-induced osteoporosis [12]. However, possible involvement of the ER stress in the development of osteoporosis has not well been understood.

The major types of osteoporosis in humans include postmenopausal osteoporosis, disuse osteoporosis, and glucocorticoid-induced osteoporosis[13-16]. Unloading in animals is often mimicked by tail suspension or sciatic neurectomy for quantitative evaluation of the progress of disuse osteoporosis[13, 17, 18]. But the mechanism underlying enhanced osteoclasts activity and impaired osteoblasts functions remains to be clarified. In the current study, the unloading model was used to analyze the pathogenesis of osteoporosis, and the relationship between ER stress and osteoporosis.

Salubrinal is a synthetic chemical (480 Da, C21H17Cl3N4OS) known to block 
the dephosphorylation of eukaryotic translation initiation factor 2 alpha (eIF2 $\alpha$ ), which plays a critical role in the responses to the ER stress[19]. The elevated phosphorylation level of eIF2 $\alpha$ activates translation of activating transcription factor 4 (ATF4), which is one of the key transcription factors in bone formation[20]. Little is known, however, on its therapeutic effects on unloading-induced osteoporosis. In the present study, the unloading model was used to analyze the pathogenesis of osteoporosis, and salubrinal was employed as an agent to evaluate the role of ER stress in disuse osteoporosis.

Using unloaded mice as a model for disuse osteoporosis, we first investigated herein the pathogenesis of osteoporosis, focusing on the role of ER stress in the development of osteoporosis. We also examined the effects of salubrinal to unloaded mice, target the development of bone marrow-derived cells that give rise to both bone-forming osteoblasts and bone-resorbing osteoclasts. 


\section{Materials and Methods}

Animals and materials preparation

One hundred and seventeen C57BL/6 female mice (Animal Center of Academy of Military Medical Sciences, China), $\sim 16$ weeks of age, were used. Four to five mice per cage were housed under pathogen-free conditions, and were fed with standard laboratory rodent chow and water ad libitum. Mice were maintained at a constant temperature of $25 \square$, and kept on a 12-hour light/dark cycle during the experimental procedure. All experiments were carried out according to the National Institutes of Health Guide for Care and Use of Laboratory Animals and were approved by the Ethics Committee of Tianjin Medical University. Murine receptor activator of nuclear factor kappa-B ligand (RANKL) and murine macrophage-colony stimulating factor (M-CSF) were purchased from PeproTech (Rocky Hills, NC, USA). Dulbecco's Modified Eagle's Medium (DMEM), Minimum Essential Medium Alpha (MEM- $\alpha$ ), fetal bovine serum, penicillin, streptomycin and trypsin were purchased from Invitrogen (Carlsbad, CA, USA). Other chemicals were purchased from Sigma (St. Louis, MO, USA) unless otherwise stated.

\section{Experimental design}

In the first set of experiments, fifty-four mice were used to evaluate the role of ER stress in the development of unloading-induced osteoporosis. These mice were divided into nine groups: the age-matched control $(\mathrm{AC} ; \mathrm{n}=6)$ and eight hindlimb unloading groups (HU). To examine the pathogenesis of disuse osteoporosis, unloaded mice were subdivided based on the duration for unloading such as $3 \mathrm{~h}, 6 \mathrm{~h}$, $12 \mathrm{~h}, 1 \mathrm{~d}, 2 \mathrm{~d}, 3 \mathrm{~d}, 7 \mathrm{~d}$, and $14 \mathrm{~d}(\mathrm{n}=6$ for each subgroup).

In the second set of experiments, forty-five mice were employed to assess the effect of salubrinal on unloading-induced osteoporosis. These mice were divided into three groups $(n=21)$ : the age-matched control group (AC), hindlimb unloading group (HU), and salubrinal-treated hindlimb unloading group (US). Thirty mice (HU and US) were subjected to hindlimb unloading for 2 weeks. All animals were weighed prior to any treatment and at sacrifice on 2 weeks. 


\section{In vivo tail suspension}

The mice were outfitted with a custom-made tail harnesses and suspended from an overhead pulley system in the customized cage. The position of the mice was adjusted to maintain in a $\sim 30^{\circ}$ head-down tilt with the hindlimbs elevated above the floor. The mice were able to ambulate within the cage using their forelimbs, which remained in contact with the cage floor. However, their hindlimbs remained suspended in air and consequently were unable to receive ground reaction forces (Fig 1A). Food and water were provided on the cage floor. For the age-matched control group, mice were housed individually under the same conditions but were not subjected to hindlimb unloading[21-23].

\section{Subcutaneous administration of salubrinal}

In the $2^{\text {nd }}$ set of experiments, for salubrinal-treated group, unloaded mice received subcutaneous injections of salubrinal (Tocris Bioscience, Ellisville, MO, USA) in propylene glycol daily at a dose of $1 \mathrm{mg} / \mathrm{kg}$ body weight for 2 weeks. The placebo mice received an equal volume of vehicle[12]

\section{Measurements of bone mineral density and bone mineral content}

The animals were anesthetized by $1.5 \%$ isoflurane at a flow rate of $1.0 \mathrm{~L} / \mathrm{min}$, placed on the platform in the prone position, and their images were acquired in $\sim 5$ min. Using peripheral dual energy X-ray absorptiometry (pDEXA; PIXImus II, Lunar Corp, Madison, WI, USA), bone mineral density (BMD, $\mathrm{g} / \mathrm{cm}^{2}$ ) and bone mineral content $(\mathrm{BMC}, \mathrm{g})$ of the bilateral femur were measured before unloading and sacrifice (version 1.47). We scanned the entire animal, and conducted ROI analysis. The changes in BMD and BMC were determined, and statistical analysis was conducted.

\section{Micro-computed tomography}

Micro-computed tomography $(\mu \mathrm{CT})$ was performed using a ScancovivaCT 40 (Scanco Medical AG, Bassersdorf, Switzerland), a high-resolution desk-top system as previously reported[24]. Briefly, the excised left distal femora were scanned using an 
X-ray source set at $60 \mathrm{kV}$ with $6-\mu \mathrm{m}$ pixel size. The trabecular bone compartment was segmented from the cortical shell for 50 slices in a region $\sim 0.5 \mathrm{~mm}$ proximal to the most distal portion of the growth plate. A three-dimensional (3D) analysis was done to determine bone volume fraction (BV/TV, \%), trabecular number (Tb.N, 1/mm), trabecular thickness (Tb.Th, $\mu \mathrm{m})$, and trabecular bone spacing (Tb.Sp, $\mu \mathrm{m})$.

\section{Histology, TRAP, MacNeal, TUNEL, and immunohistochemistry assays}

The distal femora were fixed in 10\% neutral buffered formalin for two days and decalcified in 14\% EDTA for 2 weeks. Decalcified samples were embedded in paraffin, and 5- $\mu \mathrm{m}$-thick coronal sections were cut. The slides were then processed for hematoxylin and Eosin (H\&E) staining. The images of the distal femora were captured with an Olympus BX53 microscope and Olympus DP73 camera. Measurements were performed within 1.6- $\mathrm{mm}^{2}$ sample area on the proximal side of the growth plate $(\sim 0.8 \mathrm{~mm}$ proximal distance from the growth plate), in which B.Ar/T.Ar (bone area/total area) was determined.

Tartrate resistant acid phosphatase (TRAP) staining was used to determine osteoclast development[25]. The ratio between length of TRAP-positive cells and total circumference of bone trabecula were calculated. MacNeal's staining was used for identifying osteoblasts as described previously[26, 27]. The osteoblast number was normalized using the trabecular bone surface.

For immunohistochemical analysis, femoral sections were incubated with primary antibodies against nuclear factor of activated T-cells, cytoplasmic 1 (NFATc1) (Abcam, Cambridge, MA, USA) at $4 \square$ overnight. An immunohistochemical kit and 3, 3'-diaminobenzidine (DAB) (ZSGB-BIO, Beijing, China) substrate kit were used according to the manufacturer protocol. Histomorphometric measurements were conducted on the area of the proximal femur. Quantitative analysis was conducted in a blinded fashion[28].

TUNEL assay

TUNEL staining was performed using a DeadEnd ${ }^{\mathrm{TM}}$ Fluorometric TUNEL 
System (Promega, Madison, WI, USA) and apoptotic cells from the femoral section and MC3T3-E1 cells were detected [29, 30]. Fluorescently labelled cells were identified in 5 fields of view, and the relative percentage of TUNEL positive nuclei was determined using image analysis software (Cellsense standard software).

\section{Isolation of bone marrow-derived cells for osteoclast development}

After euthanasia, bone marrow-derived cells were collected by flushing the iliac with Iscove's MEM (Gibco-Invitrogen, Carlsbad, CA, USA) that contained 2\% fetal bovine serum (FBS). Cells were separated by low-density gradient centrifugation and cultured in $\alpha$-MEM supplemented with $10 \%$ FBS, $30 \mathrm{ng} / \mathrm{ml}$ murine macrophage-colony stimulating factor (M-CSF), and $20 \mathrm{ng} / \mathrm{ml}$ murine receptor activator of nuclear factor kappa-B ligand (RANKL). On day 3, the culture medium was replaced by $\alpha$-MEM supplemented with $10 \% \mathrm{FBS}, 30 \mathrm{ng} / \mathrm{ml} \mathrm{M}-\mathrm{CSF}$, and 60 ng/ml RANKL. Cells were grown for three additional days[31, 32].

Assays for colony-forming unit-fibroblasts (CFU-F) and colony-forming unit-osteoblasts (CFU-Ob)

To evaluate colony formation capability of fibroblast-like mesenchymal stem cells, the CFU-F assay was performed. In brief, bone marrow-derived cells $\left(2 \times 10^{6}\right.$ cells $/ \mathrm{ml}$ ) were cultured in 6-well culture plates in a complete MesenCult medium. Fresh medium was exchanged every other day. On day 14, cells were stained using a HEMA-3 quick staining kit (Fisher Scientific, Waltham, MA, USA). The number of CFU-F colonies with more than 50 cells was counted, and the clusters of cells that did not present fibroblast-like morphology were excluded[24, 33, 34].

In the CFU-Ob assay, bone marrow-derived cells were plated at $2 \times 10^{6}$ cells $/ \mathrm{ml}$ in 6-well plates consisting of the osteogenic differentiation medium (MesenCult proliferation kit, supplemented with $10 \mathrm{nM}$ dexamethasone, $50 \mu \mathrm{g} / \mathrm{ml}$ ascorbic acid 2-phosphate, and $10 \mathrm{mM} \beta$-glycerophosphate) [31]. The medium was changed every other day, and cells were cultured for 2 weeks. On day 14, cells were stained using an alkaline phosphatase (ALP) kit (Sigma-Aldrich, St. Louis, MO, USA). The 
percentage of ALP-positive colonies was calculated.

Assays for Colony-forming unit-macrophage/monocyte (CFU-M) and Colony-forming unit-granulocyte-macrophages (CFU-GM)

The CFU-M and CFU-GM assays were conducted using bone marrow mononuclear cells as described previously [31, 35]. Approximately $2.5 \times 10^{4}$ bone marrow-derived cells from the iliac were prepared. Cells were seeded onto a $35-\mathrm{mm}$ gridded dish, which was composed of methylcellulose supplemented with $30 \mathrm{ng} / \mathrm{ml}$ M-CSF and $20 \mathrm{ng} / \mathrm{ml}$ RANKL. Cells were cultured for 7 days in the presence and absence of salubrinal. The colony numbers of CFU-M and CFU-GM were converted to the numbers per iliac.

\section{Assay for differentiation to mature osteoclasts}

The osteoclast differentiation assay was performed using bone marrow-derived cells from unloading group in 96-well plates in the presence and absence of salubrinal[36]. During 6-day experiments, the culture medium was exchanged once on day 4. Adherent cells were fixed and stained with a tartrate resistant acid phosphate (TRAP)-staining kit (Sigma-Aldrich, St. Louis, MO, USA). TRAP-positive multinucleated cells ( $>3$ nuclei) were identified as mature osteoclasts, and the area covered by mature osteoclasts was determined.

\section{Assays for the migration and adhesion of pre-osteoclasts}

Migration of osteoclasts was evaluated using a transwell assay as described previously with minor modifications[31]. Bone marrow-derived cells $\left(2 \times 10^{6}\right.$ cells $/ \mathrm{ml}$ in 6-well plates) were cultured in M-CSF and RANKL for 4 days. The osteoclast precursor cells $\left(1 \times 10^{5}\right.$ cells/well $)$ were loaded onto the upper chamber of transwells and allowed to migrate to the bottom chamber through an $8-\mu \mathrm{m}$ polycarbonate filter coated with vitronectin (Takara Bio Inc, Otsu, Shigma, Japan). The bottom chamber contained $\alpha$-MEM consisting of $1 \%$ bovine serum albumin (BSA) and $30 \mathrm{ng} / \mathrm{ml}$ M-CSF. After $6 \mathrm{~h}$ reaction, the number of osteoclast precursor cells in the lower 
chamber (attached onto the bottom of the transwells) was counted.

For assaying adhesion, osteoclast precursor cells $\left(1 \times 10^{5}\right.$ cells/well $)$ were placed into 96 -well plates coated with $5 \mu \mathrm{g} / \mathrm{ml}$ vitronectin in $\alpha$-MEM supplemented with 30 ng/ml M-CSF[37]. After $30 \mathrm{~min}$ of incubation, cells were washed with PBS three times and fixed with $4 \%$ paraformaldehyde at room temperature for 10-15 min. Cells were stained with crystal violet, and the number of adherent cellswas counted.

\section{Cell viability assay}

An MTT assay was used to evaluate cell viability as previously described [38]. RAW264.7 cells and MC3T3-E1 cells were seeded in 96-well plates at a density of $1 \times 10^{4}$ cells/well. Four hours later, cells were treated with the agent or vehicle (DMSO). After $48 \mathrm{~h}$, the absorbance at $570 \mathrm{~nm}$ was detected using a $\mu$ Quant universal microplate spectrophotometer (Bio-tek, Winooski, USA).

\section{Western blot analysis}

For Western blot analysis, protein samples were isolated from the femora using a mortar and pestle. Tissues were lysed in a radioimmunoprecipitation assay (RIPA) lysis buffer, containing protease inhibitors and phosphatase inhibitors (Roche Diagnostics GmbH, Mannheim, Germany). Isolated proteins were fractionated using $10 \%$ sodium dodecyl sulfate-polyacrylamide gels and electro-transferred to polyvinylidenedifluoride membranes (Millipore, Billerica, MA, USA)[28]. Primary antibodies specific to Bip (Affinity BioReagents, Suwanee, GA, USA), eIF2 $\alpha$, phospho-eIF2 $\alpha$, ATF4 (Cell Signaling, Danvers, MA, USA), RANKL, cathepsin K, CHOP (the CCAAT/enhancer binding protein homologous protein) (Proteintech, Wuhan, China) and $\beta$-actin (Sigma, St Louis, USA) were employed. After incubation with secondary $\operatorname{IgG}$ antibodies conjugated with horseradish peroxidase, signals were detected with enhanced chemiluminescence. Data were presented with reference to control intensities of $\beta$-actin. 
The data were expressed as mean \pm standard deviation (SD). Data were analyzed with independent-sample $t$ test (for two groups) or one-way ANOVA (for more than two groups). For pair-wise comparisons, a post-hoc test was conducted using Fisher's protected least significant difference. Correlation analysis was performed using Pearson correlation coefficient test. The relative parameters ( $\%$ change) such as body weight, $\mathrm{BMD}$, and $\mathrm{BMC}$ were calculated as $([\mathrm{S}-\mathrm{B}] / \mathrm{B} \times 100$ in $\%$, where $\mathrm{S}=$ "sacrifice" and B = "baseline". All comparisons were two-tailed and statistical significance was assumed at $p<0.05$. The asterisks $(*, * *$, and $* * *)$ represent $p<0.05$, $p<0.01$, and $p<0.001$, respectively. 


\section{Results}

The animals, used for tail suspension and administration of salubrinal, tolerated the procedures. No bruising or tissue damage was detected at the tail suspension site.

\section{Unloading-driven alteration in body weight and B.Ar/T.Ar in the femur}

During the 2-week experiment, age-matched control in body weight increased. However, unloaded mice demonstrated a time-dependent decrease in body weight (Fig. 1B). Compared to age-matched control, a statistically significant decrease was observed in these 4 unloaded groups (all $p<0.001$ ). Similar to the change of body weight, the histological samples of the distal femora from the unloaded mice showed a time-dependent decrease in B.Ar/T.Ar (Fig. 1C). Compared to age-matched control, a statistically significant decrease was observed in HU3d $(p<0.05)$, HU7d $(p<0.001)$, and HU14d $(p<0.001)$, no significant difference was found in HU1d $(p=0.433)$.

Unloading-driven alteration in both osteoblast number and osteoclast number in the femur

Compared to the age-matched control mice, unloaded group showed that the number of osteoblasts on bone surface was significantly decreased in a time-dependent fashion in HU1d $(p<0.05)$, HU3d $(p<0.01)$, HU7d $(p<0.01)$, and HU14d $(p<0.001)$ (Fig. 1D). However, unloaded group presented that the ratio of TRAP-positive cells to trabecular bone surface was significantly increased in a time-dependent manner in HU3d $(p<0.01)$, HU7d $(p<0.01)$, and HU14d $(p<0.001)$, whereas HU1d showed no significant increase ( $\mathrm{p}=0.335)$ (Fig. 2A).

\section{Unloading stimulated development of osteoclasts and CFU-M/CFU-GM}

Compared to the bone marrow-derived cells isolated from the age-matched control, the cells from the unloaded mice exhibited an increase in the surface area occupied by multi-nucleated osteoclasts $(p<0.001)$ (Fig. 2B). Pre-osteoclast cells isolated from the unloaded mice were more migratory than those from the age-matched control $(p<0.001)$ (Fig. 2C). In the adhesion assay, the cells isolated 
from the unloaded mice presented an increase in adhesion over those from the age-matched control $(p<0.001)$ (Fig. 2D). Furthermore, the number of CFU-M $(p<0.001$; Fig. 2E) and CFU-GM $(p<0.001$; Fig. $2 \mathrm{~F})$ in an iliac significantly increased in the unloaded group.

\section{Unloading-induced ER stress}

Compared to the age-matched control, the unloading group significantly increased the expression of Bip in HU3h $(p<0.01)$, HU6h $(p<0.05)$ and HU12h $(p<0.01)$, but decreased in HU2d, HU3d, HU7d (all $p<0.05)$, and HU14d $(p<0.01)$. Meanwhile, compared to the age-matched control, unloading significantly increased the expression of p-eIF2 $\alpha$ in HU3h and HU6h (both $p<0.05$ ), and HU12h group exhibited the same level as the age-matched control group $(p=0.126)$, but decreased in a time-dependent manner in HU1d, HU3d, HU7d, and HU14d (all $p<0.05$ ). The unloading group increased the level of ATF4 in HU3h and HU6h (both $p<0.05$ ). HU12h $(p=0.176)$ and HU1d $(p=0.053)$ exhibited no significant change to the age-matched control group, while they decreased in HU2d, HU3d, HU7d (all $p<0.05$ ), and HU14d $(p<0.01)$ in a time-dependent manner. The expression of CHOP showed a significant increase after HU12h (all $p<0.01$ ) (Fig. 3A-E).

For correlational analysis, the expression levels of p-eIF2 $\alpha$ were positively associated with osteoblastogenesis $(r=0.886$ in CFU-Ob, $y=70.25 \mathrm{x}-34.25, \mathrm{r}=$ 0.881 in N.Ob/BS, $y=34.05 x-5.216$, and $\mathrm{r}=0.869$ in B.Ar/T.Ar, $y=35.17 \mathrm{x}-14.04$; all $p<0.001)$. However, the expression levels of $\mathrm{p}$-eIF2 $\alpha$ were negatively associated with osteoclastogenesis $(r=-0.847$ in osteoclast formation, $y=-70.73 x+117.5, r=$ -0.869 in osteoclast migration, $\mathrm{y}=-701.3 \mathrm{x}+939.1, \mathrm{r}=-0.847$ in osteoclast adhesion, $\mathrm{y}=-451.0 \mathrm{x}+566.5, \mathrm{r}=-0.919$ in Oc.S/BS ${ }^{\mathrm{f}}, \mathrm{y}=-35.57 \mathrm{x}+44.85, \mathrm{r}=-0.896$ in CFU-M, $y=-56409 x+65751$, and $\mathrm{r}=-0.845$ in CFU-GM, $\mathrm{y}=-11676 \mathrm{x}+14016$; all $p<0.001$ ) (Fig. 3F).

\section{Attenuation of unloading-induced effects in salubrinal-treated mice}

Compared to age-matched control, unloading mice demonstrated a decrease in 
body weight $(p<0.001)$, while subcutaneous administration of salubrinal for 2 weeks significantly suppressed the unloading-induced decrease in body weight $(p<0.001$; Fig. 4A).

Compared to the age-matched control, the unloaded mice presented a significant reduction in $\mathrm{BMD}$ and $\mathrm{BMC}$ (both $p<0.001$ ). However, the unloaded mice treated with salubrinal exhibited a statistically significant recovery of BMD and BMC in the femur (both $p<0.05$ ) (Fig. 4 B\&C).

Micro-CT scanning at the distal femora (Fig. 4 D) indicated that the femoral BV/TV was increased from $18.7 \pm 1.9 \%$ (HU) to $24.1 \pm 1.7 \%$ (US) $(p<0.05$; Fig. 4E). The trabecular number increased from $5.16 \pm 0.41 / \mathrm{mm}$ (HU) to $6.19 \pm 0.421 / \mathrm{mm}$ (US) $(p<0.05)($ Fig. $4 \mathrm{~F})$, and the trabecular thickness of the femur was increased with salubrinal in the present study $(p<0.01$; Fig. $4 \mathrm{G})$. However, the trabecular spacing of the femur was decreased by salubrinal treatment ( $p<0.05$; Fig. $4 \mathrm{H})$.

Compared to age-matched control mice, unloaded mice presented a reduction in B.Ar/T.Ar $(p<0.001)$. However, administration of salubrinal significantly restored B.Ar/T.Ar $(p<0.001)$ (Fig. 4I).

\section{Salubrinal-driven enhancement of bone-forming osteoblasts in vivo}

Compared to the age-matched control group, unloaded mice for 2 weeks presented a significant reduction in the number of osteoblast on trabecular bone surface by MacNeal's staining $(p<0.001)$. However, two-week administration of salubrinal significantly increased the osteoblast number ( $p<0.001$; Fig. $5 \mathrm{~A})$.

\section{Salubrinal-driven inhibition of apoptosis in bone cavity}

TUNEL-positive cells at the distal femora presented a significant increase in unloaded mice compared to age-matched control mice $(p<0.001)$, whereas the salubrinal-treated unloading mice was significant decreased them compared to unloaded mice $(p<0.001$; Fig. 5B). 
Without salubrinal, the percentage of ALP positive colonies was $30.5 \pm 1.7 \%$ in $\mathrm{AC}$ and $25.6 \pm 2.2 \%$ in $\mathrm{HU}(p<0.05)$ (Fig. $5 \mathrm{C})$. Administration of salubrinal at 1 $\mathrm{mg} / \mathrm{kg}$ increased the osteoblast differentiation to $41.3 \pm 1.6 \%$ in vivo $(p<0.001)$. After administration of salubrinal at $0.5 \mu \mathrm{M}$ in vitro, the percentage of ALP-positive colonies increased 5.1\% ( $p<0.05$; Fig. 5D).

Compared to age-matched control, unloading mice presented a significant decrease in CFU-F $(p<0.01)$. Salubrinal treated unloading mice produced more CFU-F colonies when compared to the cultures established from hindlimb unloaded mice $(p<0.01$; Fig. 5E). Furthermore, administration of salubrinal at $0.5 \mu \mathrm{M}$ in vitro increased CFU-F colonies when compared to the cultures established from unloaded mice $(p<0.01$; Fig. 5F).

Salubrinal-driven inhibition of bone resorption and osteoclast development in vivo and in vitro

Compared to the age-matched control, TRAP staining of the distal femora showed that Oc.S/BS ${ }^{f}$ was significantly increased in the unloaded mice $(p<0.001)$. However, the elevated ratio in the hindlimb unloaded group was significantly suppressed by salubrinal $(p<0.001$; Fig. 6A).

Osteoclast formation was conducted using bone marrow-derived cells, the cells from the unloaded mice exhibited a significant increase in the surface area occupied by multi-nucleated osteoclasts $(p<0.001)$. However, salubrinal treated unloaded mice significantly decreased it compared to unloaded mice without salubrinal treatment $(p<0.001$; Fig. 6B). To further evaluate the effects of salubrinal on the formation of mature osteoclasts in vitro, three dosages of salubrinal $(1,2$, and $5 \mu \mathrm{M})$ were applied to the bone marrow-derived cells isolated from HU. Compared to the HU control, in vitro administration of salubrinal for a 6-day culture period resulted in a significant decrease in osteoclast formation (all $p<0.001$; Fig. 6C). To test the effects of salubrinal on the late development of osteoclasts, salubrinal was administered on days 4 to 6 . This timeline was also able to provide a significant decrease in osteoclast formation in a time- and dosage-dependent manner (all $p<0.001$ ) in $\mathrm{HU}$ (Fig. 6C). 
Osteoclast function was evaluated through migration and adhesion assays. Pre-osteoclast cells isolated from the unloaded mice were more active in migration than those from the age-matched control mice $(p<0.001)$. In addition, pre-osteoclast cells isolated from the salubrinal treated mice presented a significantly reduced migration rate compared to unloaded mice $(p<0.001$; Fig. 6D). A significant decrease in osteoclast migration was observed in a dosage-dependent manner in vitro (all $p<0.001$ for 1,2 and $5 \mu \mathrm{M}$; Fig. 6E). In M-CSF mediated adhesion, the cells isolated from the unloaded mice presented stronger adhesion than those from the age-matched control $(p<0.001)$. Compared to the cells isolated from the unloaded mice, the cells from the salubrinal-treated mice exhibited a significant reduction in osteoclast adhesion $(p<0.001$; Fig. $6 \mathrm{~F})$. In vitro analysis revealed a significant decrease in the osteoclasts adhesion in a dosage-dependent manner (all $p<0.001$ for 1,2 and $5 \mu \mathrm{M}$; Fig. 6G)

\section{Salubrinal-driven suppression in CFU-M and CFU-GM of mature osteoclasts}

The number of CFU-M and CFU-GM, representing the number of osteoclast progenitors from unloaded mice was significantly higher than that of $\mathrm{AC}$ (both $p<0.001)$. Cells derived from mice treated with salubrinal had the significantly lower numbers of CFU-M and CFU-GM (both $p<0.001$; Fig. 7A\&B). After administration of salubrinal at 1,2 , and $5 \mu \mathrm{M}$, a statistically significant dosage-dependent decrease in both CFU-M and CFU-GM was observed (both $p<0.001$; Fig. 7C\&D).

\section{Salubrinal-driven alteration in NFATc1}

Immunohistochemistry staining of NFATc1 showed the unloading-driven upregulation of osteoclasts. Specifically, NFATc1-positive cells in salubrinal-treated mice were significantly decreased $(p<0.01$; Fig. 7E).

\section{Salubrinal protects osteogenesis against ER stress in vitro and in vivo}

Tunicamycin was used as an activator of ER stress. We employed salubrinal at 0.2 to $5 \mu \mathrm{M}$, and evaluated their effects in the presence of tunicamycin. Tunicamycin 
increased viability of RAW264.7 cells at $100 \mathrm{ng} / \mathrm{ml}$, but salubrinal partially inhibited it in a dose-dependent manner (both $p<0.05$ at sal $1 \mu \mathrm{M}$ and $5 \mu \mathrm{M}$; Fig. 8A). Tunicamycin inhibited viability of MC3T3-E1 cells at $100 \mathrm{ng} / \mathrm{ml}$, while salubrinal restored the viability in a dose-dependent manner ( $p<0.05$ at sal $1 \mu \mathrm{M} ; p<0.01$ at sal 5 $\mu \mathrm{M}$; Fig. 8B). TUNEL-positive MC3T3-E1 cells were significantly increased in the presence of tunicamycin, whereas salubrinal treatment significantly decreased TUNEL positive cells (both $p<0.001$; Fig. $8 \mathrm{C}$ ).

Western blot analysis demonstrated that salubrinal increased the level of Bip, p-eIF2 $\alpha /$ IF $2 \alpha$ (both $p<0.05)$ and ATF4 $(p<0.01)$, but decreased the level of CHOP in vitro $(p<0.01)$ (Fig. 8D). Salubrinal increased the level of phosphorylated eIF2 $\alpha$ $(p<0.05 ; \mathrm{p}$-eIF $2 \alpha)$, while it decreased the level of RANKL, cathepsin K and CHOP (all $p<0.05$ ) compared to the unloaded mice in vivo (Fig. 8E). 


\section{Discussion}

This study demonstrates that the ER stress plays a critical role in the pathogenesis of disuse osteoporosis. Using the tail suspended mice as a disuse osteoporosis model, unloading-driven reduction in femoral B.Ar/T.Ar was observed in a time-dependent fashion. The number of osteoblasts on the distal femora was significantly decreased in the unloaded mice. TRAP staining showed that Oc.S/BS was increased in the unloaded mice. Unloading significantly increased the expression of Bip, p-eIF2 $\alpha$ and ATF4 within $12 \mathrm{~h}$ of tail suspension, but decreased at later time points in HU2d to HU14d. The results indicate that short-term ER stress can protect cell through increasing Bip, p-eIF2 $\alpha$ and ATF4 level, whereas long-term unloading induces apoptosis by inhibiting Bip, p-eIF2 $\alpha$ and ATF4. The expression of CHOP showed a time-dependent increase (Fig. 8F). Furthermore, to understand the role of ER stress in induction of disuse osteoporosis, the effect of salubrinal as an eIF2 $\alpha$ dephosphorylation inhibitor that block unloading-induced ER stress was investigated. Administration of salubrinal significantly suppressed unloading-induced bone loss and prevented apoptosis. Salubrinal also evaluated the levels of Bip, p-eIF2 $\alpha / \mathrm{eIF} 2 \alpha$, ATF4, RANKL, CHOP, and cathepsin $\mathrm{K}$ in tail-suspended mice. Meanwhile, administration of salubrinal in age-matched control (non-osteoporotic mice), no detectable changes were observed on bone formation and osteoclastogenesis (data not shown). These results showed that unloading-induced disuse osteoporosis modulates the expression of Bip, p-eIF2 $\alpha$, ATF4 and CHOP, indicating the possibility that the ER stress is involved in disuse osteoporosis.

Although lots of models such as ovariectomy-induced osteoporosis, glucocorticoid-induced osteoporosis, and disuse osteoporosis has been generated[13, $39,40]$, disuse osteoporosis is selected in current study since the stimulus intensity and procedure of hindlimb suspension can be easy operated and quantified analysis for the development of osteoporosis. The tail-suspended animal model has been widely accepted as an effective disuse osteoporosis model for simulating bone loss[15, $22,41]$. In the current tail suspension experiment, bone formation was mildly inhibited and bone resorption was markedly increased in unloaded mice, these 
changes are in accord with previous study[14, 42-44]. Our correlation analysis and linear regression showed that the expression level of p-eIF2 $\alpha$ was positively associated with the number of bone-forming osteoblasts, and negatively associated with that of bone-resorbing osteoclasts. Therefore, disuse osteoporosis model was used in the current study.

In the second set of experiments, salubrinal was applied as a dissecting tool to evaluate the role of ER stress in the development and treatment of osteoporosis. As shown in previous study, salubrinal inhibited eIF2 $\alpha$ dephosphorylation through ER stress in glucocorticoid induced bone loss animal model[12]. In our study, ER stress induced by unloading and was significantly inhibited by salubrinal in disuse osteoporosis. Our results showed that daily subcutaneous administration of salubrinal significantly suppressed unloading-induced decrease of bone mass. During bone remodeling progresses, osteoblasts and fibroblasts are generated from bone marrow mesenchymal stem cells [34]. To evaluate the proliferation and differentiation of these stem cells, we conducted CFU-F and CFU-Ob assays. The increase in CFU-F by salubrinal suggested stimulated proliferation of MSCs in bone marrow-derived cells, while the elevated number of ALP-positive cells in CFU-Ob as well as the osteoblast number on bone surface indicated an enhancement of osteoblast differentiation. The results of the CFU-F and $\mathrm{CFU}-\mathrm{Ob}$ assays are consistent with salubrinal-driven augmentation of bone formation in the unloaded mice. Subcutaneous injection of salubrinal as well as in vitro suppressed the maturation of osteoclasts, the migration and adhesion of pre-osteoclasts, as well as the population of colony-forming unit-macrophage. The result shows that salubrinal is able to prevent bone loss in disuse osteoporosis and induce the promotion of osteoblastogenesis as well as the suppression of osteoclastogenesis.

Our study consistent with salubrinal-driven augmentation of bone formation in the unloaded mice by decreasing ER stress, inhibiting eIF2 $\alpha$ dephosphorylation by salubrinal prevents osteoblast apoptosis, accelerate the healing of bone wounds[45], enhance bone formation[12], and increase the mineralization of MC3T3 cells[32, 46]. The elevated level of p-eIF $2 \alpha$ contributes to osteoblast differentiation in disuse 
osteoporosis by the suppression of cellular stress, such as stress to the ER and radiation, through decreasing translational efficiency in general, except for a set of proteins such as ATF4[47]. Cell viability and TUNEL assays were conducted to evaluate apoptotic cells. In histological TUNEL assay in vivo, apoptotic cells were located in bone marrow cavity. For in vitro assay, ER stress induced osteoblast apoptosis, and salubrinal rescued it. However, osteoclast apoptosis was not observed in RAW264.7 cells.

The development of osteoclasts was suppressed by both subcutaneous injection of salubrinal as well as in vitro incubation of bone marrow-derived cells with salubrinal[32, 48]. Although the effects of salubrinal in RAKNL-induced osteoporosis were reported[32], its therapy on disuse model that is one of clinical related osteoporosis has not been validated. Importantly, salubrinal suppressed the maturation of osteoclasts in both early and late development phases, the migration and adhesion of pre-osteoclasts. Furthermore, macrophage (G) and mononuclear (M) were osteoclast progenitors [25]. Salubrinal affected the development of osteoclasts in osteoporosis by CFU-M/CFU-GM assays. NFATc1 is usually expressed in osteoclasts, and is linked to osteoclastogenesis. Our previous studies have demonstrated that salubrinal suppresses osteoclastogenesis by reducing NFATc1 $[28,46]$. The result for NFATc1 in this study is consistent with those previous studies. The osteoclast formation is associated with osteoclast-specific genes including RANKL and NFATc1,as well as ER stress[49]. Because of the role of Bip and phosphorylated eIF $2 \alpha$ in reducing translational efficiency, the suppression of NFATc 1 by salubrinal is likely to be regulated at least in part at the level of translation [50]. Osteoclastogenesis by unloading were reduced by salubrinal through regulation of Bip, eIF $2 \alpha$ and NFATc1 (Fig. 8G).

PERK (double-stranded RNA-activated protein kinase-like ER kinase) is one of the three principal kinases in the unfolded protein response (UPR) in ER stress. When activated, PERK phosphorylates eIF2 $\alpha$ and inhibits general translational activities. In this way, an increase in p-eIF $2 \alpha$ reduces the flux of proteins entering into the ER [1]. In our study, we demonstrated that p-eIF $2 \alpha /$ IF $2 \alpha$ was elevated in response to 
short-term ER stress. For long-term stress, CCAAT/enhancer CHOP was elevated. CHOP regulates expression of pro-apoptotic factors and blocks BCL-2. Hence, CHOP has the propensity to drive apoptosis [51] and thus cells might be led to apoptosis. Our linear regression analysis showed that the expression level of p-eIF2 $\alpha$ was positively associated with the number of bone-forming osteoblasts, and negatively associated with that of bone-resorbing osteoclasts.

In summary, this study demonstrates the crucial role of ER stress in the pathogenesis of osteoporosis using disuse osteoporosis model. The results herein utilizing in vivo of the unloaded mouse model and in vitro analysis of primary bone marrow-derived cells, reveal that administration of salubrinal is effective in attenuating apoptosis, as well as stimulating osteoblastogenesis and inhibiting osteoclastogenesis by suppressing dephosphorylation of eIF2 $\alpha$. The current experiments also provide the possibility of salubrinal as a therapeutic agent for reversing bone loss from osteoporosis by suppressing unloading-associated ER stress. Further analysis will target the molecular mechanism of salubrinal's action warrants the potential development of a novel strategy for combating unloading-driven osteoporosis through eIF $2 \alpha$ signaling. 


\section{Acknowledgements}

This study was supported by grants from the National Natural Science Foundation of China (81572100 to PZ), the Tianjin Municipal Science and Technology Commission (14JCZDJC36500 to PZ). 


\section{References}

[1] P. Walter, D. Ron, The unfolded protein response: from stress pathway to homeostatic regulation, Science 334(6059) (2011) 1081-6.

[2] D.T. Rutkowski, R.J. Kaufman, A trip to the ER: coping with stress, Trends Cell Biol 14(1) (2004) 20-8.

[3] P. van Galen, A. Kreso, N. Mbong, D.G. Kent, T. Fitzmaurice, J.E. Chambers, S. Xie, E. Laurenti, K. Hermans, K. Eppert, S.J. Marciniak, J.C. Goodall, A.R. Green, B.G. Wouters, E. Wienholds, J.E. Dick, The unfolded protein response governs integrity of the haematopoietic stem-cell pool during stress, Nature 510(7504) (2014) 268-72.

[4] L. Salvado, X. Palomer, E. Barroso, M. Vazquez-Carrera, Targeting endoplasmic reticulum stress in insulin resistance, Trends Endocrinol Metab 26(8) (2015) 438-48.

[5] R. Ghosh, L. Wang, E.S. Wang, B.G. Perera, A. Igbaria, S. Morita, K. Prado, M. Thamsen, D. Caswell, H. Macias, K.F. Weiberth, M.J. Gliedt, M.V. Alavi, S.B. Hari, A.K. Mitra, B. Bhhatarai, S.C. Schurer, E.L. Snapp, D.B. Gould, M.S. German, B.J. Backes, D.J. Maly, S.A. Oakes, F.R. Papa, Allosteric inhibition of the IRE1alpha RNase preserves cell viability and function during endoplasmic reticulum stress, Cell 158(3) (2014) 534-48.

[6] C.Y. Chung, V. Khurana, P.K. Auluck, D.F. Tardiff, J.R. Mazzulli, F. Soldner, V. Baru, Y. Lou, Y. Freyzon, S. Cho, A.E. Mungenast, J. Muffat, M. Mitalipova, M.D. Pluth, N.T. Jui, B. Schule, S.J. Lippard, L.H. Tsai, D. Krainc, S.L. Buchwald, R. Jaenisch, S. Lindquist, Identification and rescue of alpha-synuclein toxicity in Parkinson patient-derived neurons, Science 342(6161) (2013) 983-7.

[7] T.S. Lisse, F. Thiele, H. Fuchs, W. Hans, G.K. Przemeck, K. Abe, B. Rathkolb, L. Quintanilla-Martinez, G. Hoelzlwimmer, M. Helfrich, E. Wolf, S.H. Ralston, M. Hrabe de Angelis, ER stress-mediated apoptosis in a new mouse model of osteogenesis imperfecta, PLoS Genet 4(2) (2008) e7.

[8] J.A. Kanis, E.V. McCloskey, N.C. Harvey, H. Johansson, W.D. Leslie, Intervention Thresholds and the Diagnosis of Osteoporosis, J Bone Miner Res 30(10) (2015) 1747-53.

[9] D. Nih Consensus Development Panel on Osteoporosis Prevention, Therapy, Osteoporosis prevention, diagnosis, and therapy, JAMA 285(6) (2001) 785-95.

[10] J.Y. Kim, Y.H. Cheon, S.C. Kwak, J.M. Baek, K.H. Yoon, M.S. Lee, J. Oh, Emodin regulates bone remodeling by inhibiting osteoclastogenesis and stimulating osteoblast formation, J Bone Miner Res 29(7) (2014) 1541-53.

[11] S. Hino, S. Kondo, K. Yoshinaga, A. Saito, T. Murakami, S. Kanemoto, H. Sekiya, K. Chihara, Y. Aikawa, H. Hara, T. Kudo, T. Sekimoto, T. Funamoto, E. Chosa, K. Imaizumi, Regulation of ER molecular chaperone prevents bone loss in a murine model for osteoporosis, J Bone Miner Metab 28(2) (2010) 131-8.

[12] A.Y. Sato, X. Tu, K.A. McAndrews, L.I. Plotkin, T. Bellido, Prevention of glucocorticoid induced-apoptosis of osteoblasts and osteocytes by protecting against endoplasmic reticulum (ER) stress in vitro and in vivo in female mice, Bone 73 (2015) 60-8.

[13] T. Komori, Animal models for osteoporosis, Eur J Pharmacol 759 (2015) 287-94.

[14] S.M. Uddin, Y.X. Qin, Dynamic acoustic radiation force retains bone structural and mechanical integrity in a functional disuse osteopenia model, Bone 75 (2015) 8-17.

[15] P. Zhang, K. Hamamura, H. Yokota, A brief review of bone adaptation to unloading, Genomics Proteomics Bioinformatics 6(1) (2008) 4-7.

[16] M.P. Akhter, G.K. Alvarez, D.M. Cullen, R.R. Recker, Disuse-related decline in trabecular bone structure, Biomech Model Mechanobiol 10(3) (2011) 423-9.

[17] S. Moriya, Y. Izu, S. Arayal, M. Kawasaki, K. Hata, C. Pawaputanon Na Mahasarakhahm, Y. Izumi, P. 
Saftig, K. Kaneko, M. Noda, Y. Ezura, Cathepsin K Deficiency Suppresses Disuse-Induced Bone Loss, J Cell Physiol 231(5) (2016) 1163-70.

[18] A.C. Aryal, K. Miyai, T. Hayata, T. Notomi, T. Nakamoto, T. Pawson, Y. Ezura, M. Noda, Nck1 deficiency accelerates unloading-induced bone loss, J Cell Physiol 228(7) (2013) 1397-403.

[19] M. Boyce, K.F. Bryant, C. Jousse, K. Long, H.P. Harding, D. Scheuner, R.J. Kaufman, D. Ma, D.M. Coen, D. Ron, J. Yuan, A selective inhibitor of elF2alpha dephosphorylation protects cells from ER stress, Science 307(5711) (2005) 935-9.

[20] X. Yang, K. Matsuda, P. Bialek, S. Jacquot, H.C. Masuoka, T. Schinke, L. Li, S. Brancorsini, P. Sassone-Corsi, T.M. Townes, A. Hanauer, G. Karsenty, ATF4 is a substrate of RSK2 and an essential regulator of osteoblast biology; implication for Coffin-Lowry Syndrome, Cell 117(3) (2004) 387-98.

[21] S.M. Durbin, J.R. Jackson, M.J. Ryan, J.C. Gigliotti, S.E. Alway, J.C. Tou, Resveratrol supplementation preserves long bone mass, microstructure, and strength in hindlimb-suspended old male rats, J Bone Miner Metab 32(1) (2014) 38-47.

[22] D. Jing, J. Cai, Y. Wu, G. Shen, F. Li, Q. Xu, K. Xie, C. Tang, J. Liu, W. Guo, X. Wu, M. Jiang, E. Luo, Pulsed electromagnetic fields partially preserve bone mass, microarchitecture, and strength by promoting bone formation in hindlimb-suspended rats, J Bone Miner Res 29(10) (2014) 2250-61.

[23] J.R. Milstead, S.J. Simske, T.A. Bateman, Spaceflight and hindlimb suspension disuse models in mice, Biomed Sci Instrum 40 (2004) 105-10.

[24] S.D. Rhodes, X. Wu, Y. He, S. Chen, H. Yang, K.W. Staser, J. Wang, P. Zhang, C. Jiang, H. Yokota, R. Dong, X. Peng, X. Yang, S. Murthy, M. Azhar, K.S. Mohammad, M. Xu, T.A. Guise, F.C. Yang, Hyperactive transforming growth factor-beta1 signaling potentiates skeletal defects in a neurofibromatosis type 1 mouse model, J Bone Miner Res 28(12) (2013) 2476-89.

[25] D. Liu, X. Li, J. Li, J. Yang, H. Yokota, P. Zhang, Knee loading protects against osteonecrosis of the femoral head by enhancing vessel remodeling and bone healing, Bone 81 (2015) 620-31.

[26] R. Sharma, X. Wu, S.D. Rhodes, S. Chen, Y. He, J. Yuan, J. Li, X. Yang, X. Li, L. Jiang, E.T. Kim, D.A. Stevenson, D. Viskochil, M. Xu, F.C. Yang, Hyperactive Ras/MAPK signaling is critical for tibial nonunion fracture in neurofibromin-deficient mice, Hum Mol Genet 22(23) (2013) 4818-28.

[27] S. Huang, P.P. Eleniste, K. Wayakanon, P. Mandela, B.A. Eipper, R.E. Mains, M.R. Allen, A. Bruzzaniti, The Rho-GEF Kalirin regulates bone mass and the function of osteoblasts and osteoclasts, Bone 60 (2014) 235-45.

[28] X. Li, J. Yang, D. Liu, J. Li, K. Niu, S. Feng, H. Yokota, P. Zhang, Knee loading inhibits osteoclast lineage in a mouse model of osteoarthritis, Sci Rep 6 (2016) 24668.

[29] M.E. McGee-Lawrence, E.W. Bradley, A. Dudakovic, S.W. Carlson, Z.C. Ryan, R. Kumar, M. Dadsetan, M.J. Yaszemski, Q. Chen, K.N. An, J.J. Westendorf, Histone deacetylase 3 is required for maintenance of bone mass during aging, Bone 52(1) (2013) 296-307.

[30] T. Moriishi, Y. Kawai, H. Komori, S. Rokutanda, Y. Eguchi, Y. Tsujimoto, I. Asahina, T. Komori, Bcl2 deficiency activates FoxO through Akt inactivation and accelerates osteoblast differentiation, PLoS One 9(1) (2014) e86629.

[31] H. Yokota, K. Hamamura, A. Chen, T.R. Dodge, N. Tanjung, A. Abedinpoor, P. Zhang, Effects of salubrinal on development of osteoclasts and osteoblasts from bone marrow-derived cells, BMC Musculoskelet Disord 14 (2013) 197.

[32] L. He, J. Lee, J.H. Jang, K. Sakchaisri, J. Hwang, H.J. Cha-Molstad, K.A. Kim, I.J. Ryoo, H.G. Lee, S.O. Kim, N.K. Soung, K.S. Lee, Y.T. Kwon, R.L. Erikson, J.S. Ahn, B.Y. Kim, Osteoporosis regulation by salubrinal through elF2alpha mediated differentiation of osteoclast and osteoblast, Cell Signal 25(2) 
(2013) 552-60.

[33] Y. Li, S. Chen, J. Yuan, Y. Yang, J. Li, J. Ma, X. Wu, M. Freund, K. Pollok, H. Hanenberg, W.S. Goebel, F.C. Yang, Mesenchymal stem/progenitor cells promote the reconstitution of exogenous hematopoietic stem cells in Fancg-/- mice in vivo, Blood 113(10) (2009) 2342-51.

[34] L. Xian, X. Wu, L. Pang, M. Lou, C.J. Rosen, T. Qiu, J. Crane, F. Frassica, L. Zhang, J.P. Rodriguez, J. Xiaofeng, Y. Shoshana, X. Shouhong, E. Argiris, W. Mei, C. Xu, Matrix IGF-1 maintains bone mass by activation of mTOR in mesenchymal stem cells, Nat Med 18(7) (2012) 1095-101.

[35] H.E. Broxmeyer, F. Kappes, N. Mor-Vaknin, M. Legendre, J. Kinzfogl, S. Cooper, G. Hangoc, D.M. Markovitz, DEK regulates hematopoietic stem engraftment and progenitor cell proliferation, Stem Cells Dev 21(9) (2012) 1449-54.

[36] S.H. Mun, H.Y. Won, P. Hernandez, H.L. Aguila, S.K. Lee, Deletion of CD74, a putative MIF receptor, in mice enhances osteoclastogenesis and decreases bone mass, J Bone Miner Res 28(4) (2013) 948-59.

[37] G. Xiao, H. Cheng, H. Cao, K. Chen, Y. Tu, S. Yu, H. Jiao, S. Yang, H.J. Im, D. Chen, J. Chen, C. Wu, Critical role of filamin-binding LIM protein 1 (FBLP-1)/migfilin in regulation of bone remodeling, J Biol Chem 287(25) (2012) 21450-60.

[38] Y.X. Li, D.Q. Liu, C. Zheng, S.Q. Zheng, M. Liu, X. Li, H. Tang, miR-200a modulate HUVECs viability and migration, IUBMB Life 63(7) (2011) 553-9.

[39] A.L. Anbinder, R.M. Moraes, G.M. Lima, F.E. Oliveira, D.R. Campos, R.D. Rossoni, L.D. Oliveira, J.C. Junqueira, Y. Ma, F. Elefteriou, Periodontal disease exacerbates systemic ovariectomy-induced bone loss in mice, Bone 83 (2016) 241-7.

[40] W. Yao, W. Dai, L. Jiang, E.Y. Lay, Z. Zhong, R.O. Ritchie, X. Li, H. Ke, N.E. Lane, Sclerostin-antibody treatment of glucocorticoid-induced osteoporosis maintained bone mass and strength, Osteoporos Int 27(1) (2016) 283-94.

[41] Y. Shirazi-Fard, R.A. Anthony, A.T. Kwaczala, S. Judex, S.A. Bloomfield, H.A. Hogan, Previous exposure to simulated microgravity does not exacerbate bone loss during subsequent exposure in the proximal tibia of adult rats, Bone 56(2) (2013) 461-73.

[42] J.S. Thomsen, L.L. Christensen, J.B. Vegger, J.R. Nyengaard, A. Bruel, Loss of bone strength is dependent on skeletal site in disuse osteoporosis in rats, Calcif Tissue Int 90(4) (2012) 294-306.

[43] P. Cabahug-Zuckerman, D. Frikha-Benayed, R.J. Majeska, A. Tuthill, S. Yakar, S. Judex, M.B. Schaffler, Osteocyte Apoptosis Caused by Hindlimb Unloading is Required to Trigger Osteocyte RANKL Production and Subsequent Resorption of Cortical and Trabecular Bone in Mice Femurs, J Bone Miner Res (2016).

[44] Y. Wang, W. Liu, R. Masuyama, R. Fukuyama, M. Ito, Q. Zhang, H. Komori, T. Murakami, T. Moriishi, T. Miyazaki, R. Kitazawa, C.A. Yoshida, Y. Kawai, S. Izumi, T. Komori, Pyruvate dehydrogenase kinase 4 induces bone loss at unloading by promoting osteoclastogenesis, Bone 50(1) (2012) 409-19.

[45] P. Zhang, Q. Sun, C.H. Turner, H. Yokota, Knee loading accelerates bone healing in mice, J Bone Miner Res 22(12) (2007) 1979-87.

[46] K. Hamamura, N. Tanjung, H. Yokota, Suppression of osteoclastogenesis through phosphorylation of eukaryotic translation initiation factor 2 alpha, J Bone Miner Metab 31(6) (2013) 618-28.

[47] S. Nakamura, H. Miki, S. Kido, A. Nakano, M. Hiasa, A. Oda, H. Amou, K. Watanabe, T. Harada, S. Fujii, K. Takeuchi, K. Kagawa, S. Ozaki, T. Matsumoto, M. Abe, Activating transcription factor 4, an ER stress mediator, is required for, but excessive ER stress suppresses osteoblastogenesis by bortezomib, Int J Hematol 98(1) (2013) 66-73.

[48] K. Hamamura, A. Chen, H. Yokota, Enhancement of osteoblastogenesis and suppression of 
osteoclastogenesis by inhibition of de-phosphorylation of eukaryotic translation initiation factor 2 alpha, Receptors Clin Investig 2(1) (2015).

[49] E.G. Lee, M.S. Sung, H.G. Yoo, H.J. Chae, H.R. Kim, W.H. Yoo, Increased RANKL-mediated osteoclastogenesis by interleukin-1beta and endoplasmic reticulum stress, Joint Bone Spine 81(6) (2014) 520-6.

[50] K. Hamamura, A. Chen, N. Tanjung, S. Takigawa, A. Sudo, H. Yokota, In vitro and in silico analysis of an inhibitory mechanism of osteoclastogenesis by salubrinal and guanabenz, Cell Signal 27(2) (2015) 353-62.

[51] F. Binet, P. Sapieha, ER Stress and Angiogenesis, Cell Metab 22(4) (2015) 560-75. 


\section{Figure legends}

Fig. 1. Effects of hindlimb unloading on body weight, B.Ar/T.Ar and osteoblast number. (A) Mouse hindlimb suspension $(\mathrm{Bar}=2 \mathrm{~cm}$ ). (B) The percentage change of body weight. Loss in body weight by hindlimb suspension on day 1, 3, 7, and 14, respectively. (C) Histological parameters of trabecular bone on the proximal side of the growth plate in the distal femur were determined by H\&E staining $(\operatorname{Bar}=200 \mu \mathrm{m})$. The unloaded mice exhibited a time-dependent decrease in B.Ar/T.Ar. The representative photographs of distal femur were used to evaluate B.Ar/T.Ar. Trabecular bones were indicated by the arrows. (D) MacNeal's staining was used to determine the number of osteoblasts in the trabecular bone surface in the distal metaphysis of the femur. Hindlimb suspension showed a time-dependent decrease in the osteoblast number. The representative photographs of the distal femur were used to evaluate $\mathrm{N} . \mathrm{Ob} / \mathrm{BS} / \mathrm{mm}(\mathrm{Bar}=50 \mu \mathrm{m})$. Osteoblasts, located on the trabecular bone surface, were indicated by the arrows. The asterisks $\left(^{*}, * *\right.$, and $\left.* * *\right)$ represent statistical significance at $p<0.05, p<0.01$, and $p<0.001$, respectively $(\mathrm{n}=6)$. AC: age-matched control. HU: hindlimb unloading.

Fig. 2. Effects of hindlimb unloading on osteoclast development. (A) TRAP staining was used to evaluate bone resorption in the distal metaphysis of the femur. The representative photographs are shown on the bottom $(\mathrm{Bar}=200 \mu \mathrm{m}$ on the upper, and Bar $=50 \mu \mathrm{m}$ on the bottom). TRAP staining showed that the ratio of the number of TRAP-positive cells was time-dependent increase in the hindlimb unloaded groups. TRAP-positive cells, red color, indicated by the arrows. (B) Unloading stimulated osteoclast formation. The microphotographs on the bottom represent osteoclast cultures with TRAP staining for the age-matched control and hindlimb unloaded mice (1 week). Bar $=200 \mu \mathrm{m}$. (C-D) Effects of hindlimb unloading on osteoclast migration (C) and adhesion (D). The unloaded group significantly activated osteoclast migration and adhesion. The representative photographs are shown on the bottom. Bar $=200 \mu \mathrm{m}$. (E-F) Effects of hindlimb unloading on CFU-M (E) and CFU-GM (F). Unloading-induced increase in both CFU-M and CFU-GM in the unloaded mice. The 
images on the bottom exhibited 2 different groups, in which the circles indicate the colonies of CFU-M and CFU-GM. Bar $=500 \mu \mathrm{m}$. The asterisks (**, and ***) represent statistical significance at $p<0.01$, and $p<0.001$, respectively $(\mathrm{n}=6)$.

Fig. 3. The role of ER stress in the pathogenesis of disuse osteoporosis. (A-E) To investigate the role of ER stress in the pathogenesis of osteoporosis, p-eIF2 $\alpha / \operatorname{eIF} 2 \alpha$ was evaluated by immunoblotting. Compared to the age-matched control, the unloading group significantly increased the expression of Bip, p-eIF2 $\alpha$ and ATF4 in short-term, but decreased in a time-dependent manner in long-term. The expression of CHOP showed a significantly increase in a time-dependent manner $(n=6)$. $(F)$ Correlations between ER stress and the differentiation of osteoblasts and osteoclasts. The expression levels of p-eIF $2 \alpha$ were positively associated with bone-forming osteoblasts (CFU-Ob, N.Ob/BS, and B.Ar/T.Ar), and negatively associated with bone-resorbing osteoclasts (osteoclast development, CFU-M, and CFU-GM). The asterisks $(*)$ represent statistical significance at $p<0.05$.

Fig. 4. Effects of hindlimb unloading and salubrinal on body weight, microarchitecture, BMD, and BMC. (A) Salubrinal-driven suppression of unloading-induced loss of body weight. (B-C) Salubrinal-driven partial restore of unloading-induced loss of BMD (B) and BMC (C) in the femur. (D-G) Representative $\mu \mathrm{CT}$ reconstructed femora in the longitudinal (top) and transverse (bottom) cross-sections after 2 weeks treatment with salubrinal (D), salubrinal-driven suppression of unloading-induced loss of femoral BV/TV (E), femoral trabecular number (Tb.N) (F), and femoral trabecular thickness (Tb.Th) (G). (H) Salubrinal-driven restore of unloading-induced increase in femoral trabecular spacing (Tb.Sp). (I) Histological parameters of trabecular bone on the proximal side of the growth plate in the distal femur were determined by H\&E staining. The unloading group exhibited a lower ratio of B.Ar/T.Ar, and salubrinal enhanced B.Ar/T.Ar of the femur. The representative photographs of the distal femur are shown on the right (Bar $=500 \mu \mathrm{m})$. Trabecular bones were indicated by the arrows. The asterisks $(*, * *$, and 
***) represent statistical significance at $p<0.05, p<0.01$, and $p<0.001$, respectively (n $=15)$. US: salubrinal-treated hindlimb unloading.

Fig. 5. Effects of salubrinal on osteoblast differentiation, apoptosis, and colony forming unit-fibroblast. (A) Salubrinal-induced increase in the osteoblast numbers in unloaded mice. The microphotographs represent the three groups of MacNeal's staining $($ Bar $=50 \mu \mathrm{m})$. Osteoblasts, located on the trabecular bone surface, were indicated by the arrows. (B) DeadEnd ${ }^{\mathrm{TM}}$ Fluorometric TUNEL System was used in the distal femur to detect apoptosis. Salubrinal-driven inhibition of unloading-induced apoptosis was observed in unloaded mice. The microphotographs represent the three groups of TUNEL staining $($ Bar $=200 \mu \mathrm{m})$. Apoptotic cells were indicated by the arrows. (C) Comparison of CFU-Ob. (D) Effects of in vitro $0.5 \mu$ Msalubrinal administration on the osteoblast differentiation. (E) Comparison of CFU-F. (F) Effects of in vitro $0.5 \mu$ Msalubrinal administration on the CFU-F. The representative images are shown on the bottom in C-F. The asterisks $(*, * *$, and $* * *)$ represent statistical significance at $p<0.05, p<0.01$ and $p<0.001$, respectively $(\mathrm{n}=15)$.

Fig. 6. Effects of in vivo and in vitrosalubrinal administration on osteoclast development. (A) The osteoclast number in the unloaded group was significantly suppressed by salubrinal injection. The representative photographs are shown on the right $(\mathrm{Bar}=200 \mu \mathrm{m})$. TRAP-positive cells in the distal metaphysis of the femur, red color, indicated by the arrows. (B) Suppression of osteoclast formation by salubrinalin vivo. The microphotographs on the bottom represent the three groups of osteoclast cultures with TRAP staining. Bar $=200 \mu \mathrm{m}$. (C) Effects of in vitrosalubrinal administration on the formation of mature osteoclasts. Salubrinal was administered at 3 dosages $(1,2$, and $5 \mu \mathrm{M})$ for days $0-6$ or days $4-6$ to bone marrow-derived cells harvested from unloaded mice, and salubrinal significant decrease in the surface area of osteoclasts in a time- and dosage-dependent manner in both experiments. Four pairs of images on the bottom showed representative osteoclasts stained with TRAP. (Bar $=200 \mu \mathrm{m})$. (D) Salubrinal-induced reduction in the migration of osteoclasts in 
unloading-derived cells. (E) In vitro study showed that a significant decrease in the osteoclasts migration was observed in a dosage-dependent manner $(1,2$ and $5 \mu \mathrm{M})$. (F) Salubrinal reduced an unloading-induced increase in osteoclast adhesion. (G) In vitro study also demonstrated that a significant decrease in the osteoclasts adhesion in a dosage-dependent manner $(1,2$ and $5 \mu \mathrm{M})$. The representative photographs are shown on the bottom $(\mathrm{Bar}=200 \mu \mathrm{m}$ in $\mathrm{D}-\mathrm{G})$. Asterisk $(* * *)$ represents statistical significance at $p<0.001(\mathrm{n}=15)$.

Fig. 7. Effects of salubrinal administration on CFU-M and CFU-GM. (A-B) Salubrinal-induced reduction in CFU-M (A) and CFU-GM (B) numbers in the unloading mice. The images on the bottom exhibited the 3 different culture groups, in which the circles indicated the colonies. Bar $=500 \mu \mathrm{m}$. (C-D) In vitro administration of salubrinal at 1,2 , and $5 \mu \mathrm{M}$ in unloading-derived cells, a statistically significant dosage-dependent decrease in CFU-M (C) and CFU-GM (D) was observed. The representative photographs are shown on the bottom $($ Bar $=500 \mu \mathrm{m})$. (E) Immunohistochemistry staining and quantification of NFATc1 in distal femur. The representative photographs are shown on the right. NFATc1-positive cells in red circle. $\operatorname{Bar}=100 \mu \mathrm{m}$. Asterisk $(* * *)$ represents statistical significance at $p<0.001(\mathrm{n}=15)$.

Fig. 8. Effects of salubrinal on ER stress in vitro and in vivo. (A-B) MTT assay for cell viability of RAW264.7 cells and MC3T3-E1 cells, respectively. (C) Representative immunofluorescence images of MC3T3-E1 cells from different groups (blue: DAPI, green: $\mathrm{TUNEL}^{+}$cells, $200 \times, \mathrm{Bar}=100 \mu \mathrm{m}$ ). The numbers of $\mathrm{TUNEL}^{+}$ cells were analyzed at the bottom. (D-E) Representative images of Western blotting for salubrinal's protection of osteogenesis against the ER stress. The levels are shown on the right. The experiment was conducted in triplicate. The asterisks $(*, * *$ and $* * *)$ represent $p<0.05, p<0.01$, and $p<0.001$, respectively (S: Salubrinal, and Tm: Tunicamycin). (F) Proposed role of the ER stress in the pathogenesis of osteoporosis. (G) Mechanism of salubrinal that stimulates p-eIF2 $\alpha$ and suppresses osteoclastogenesis and osteoblast apoptosis. 
A

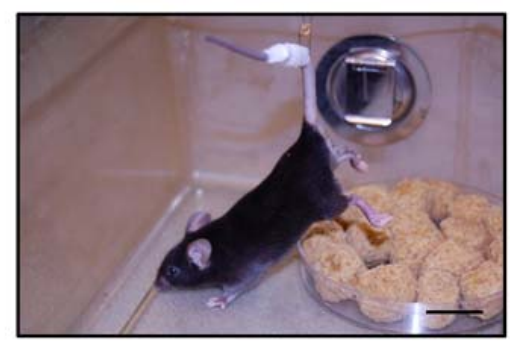

B

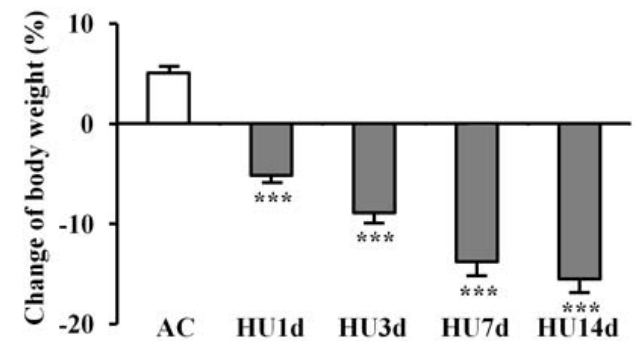

C
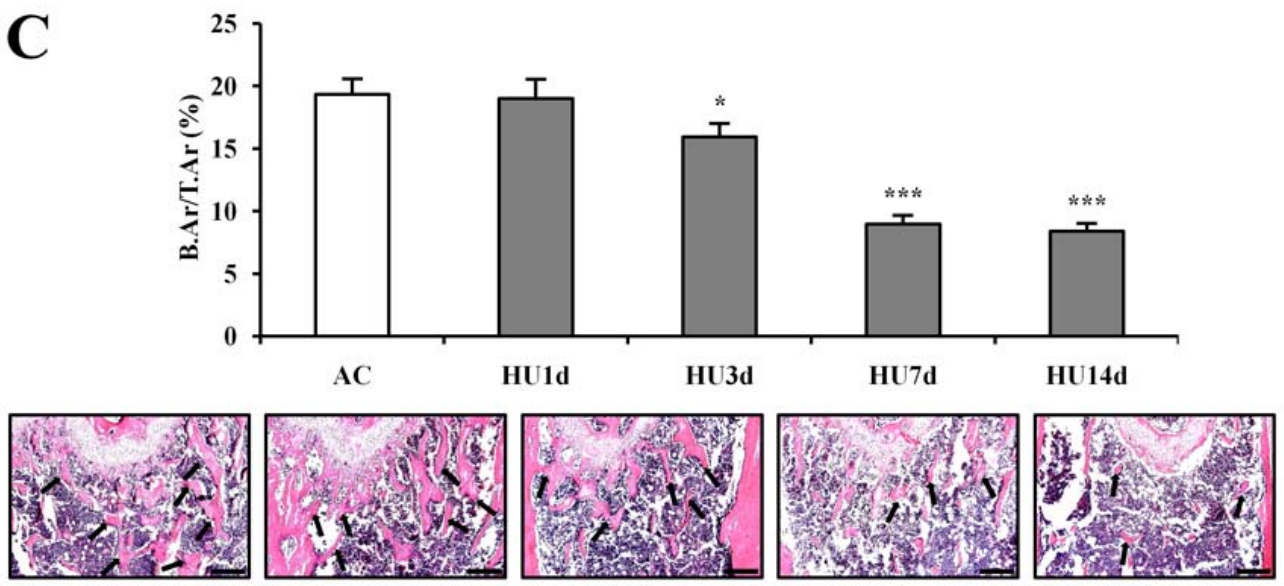

D
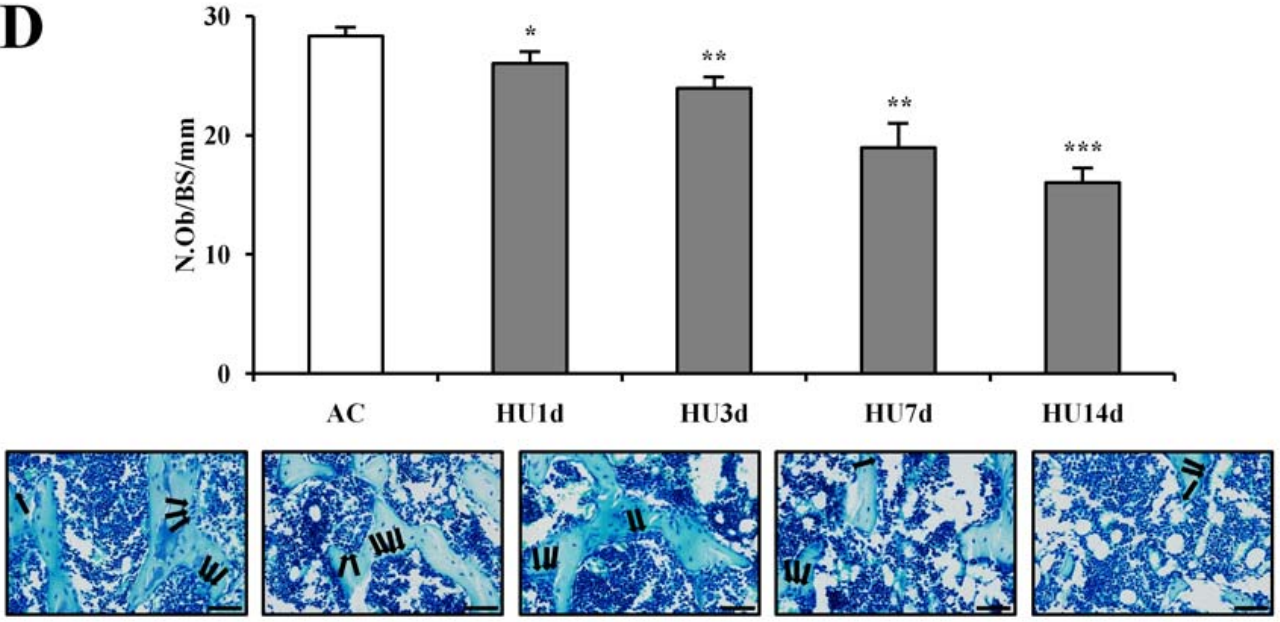

Figure 1 

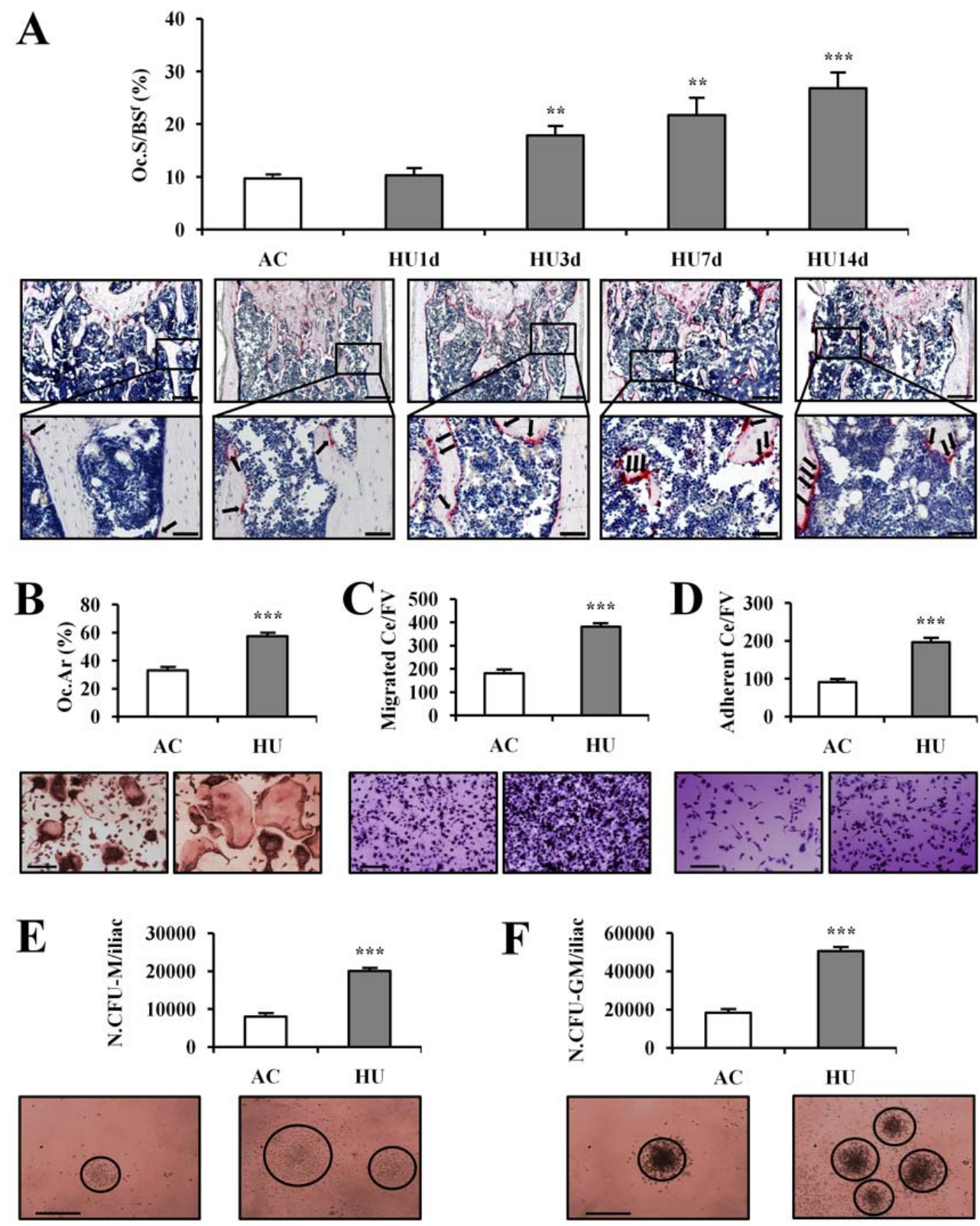

Figure 2 

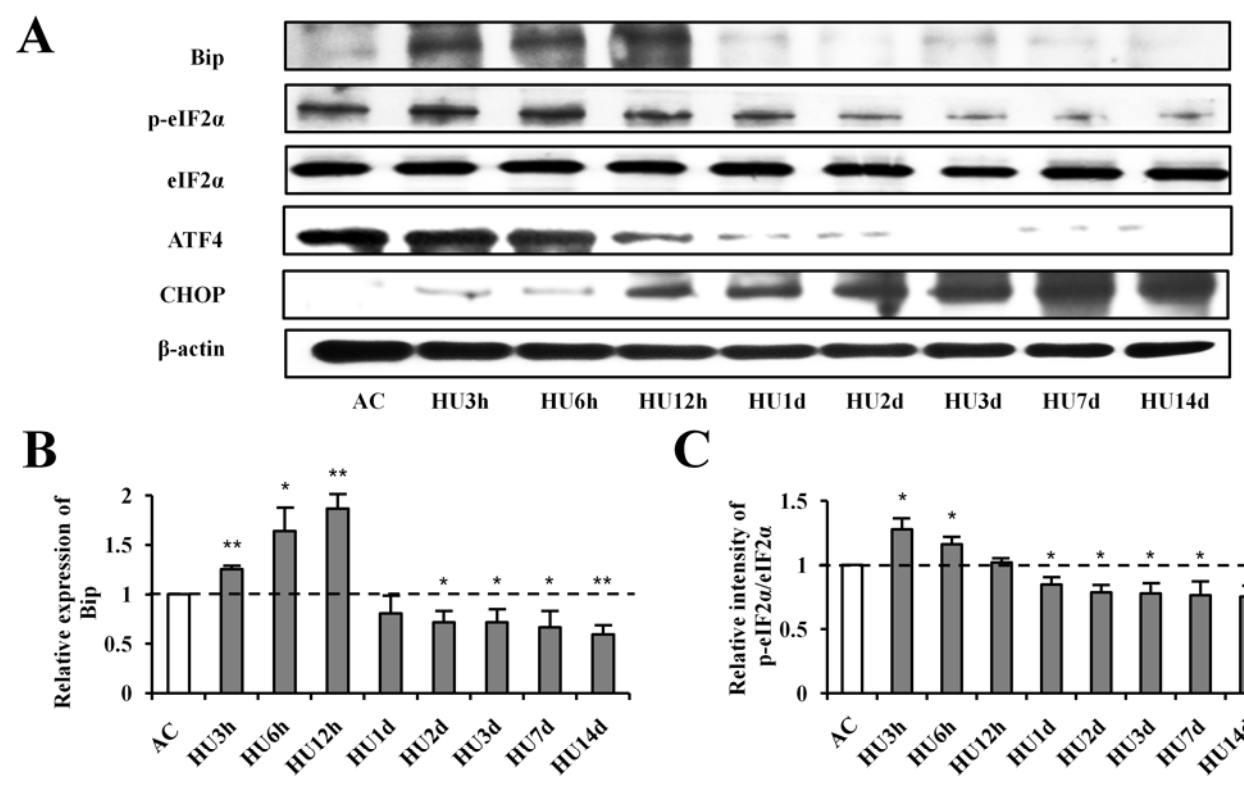

C

D
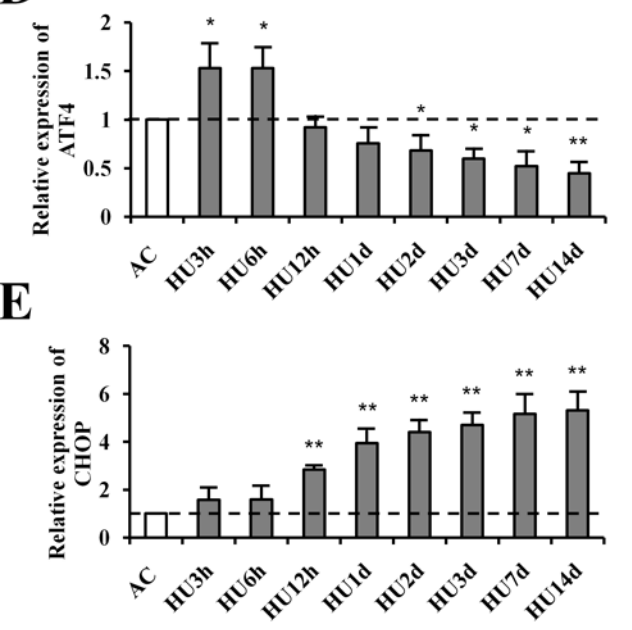

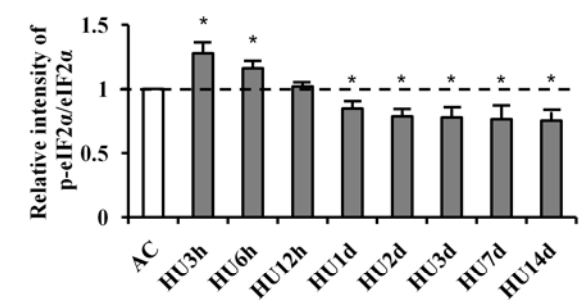

F
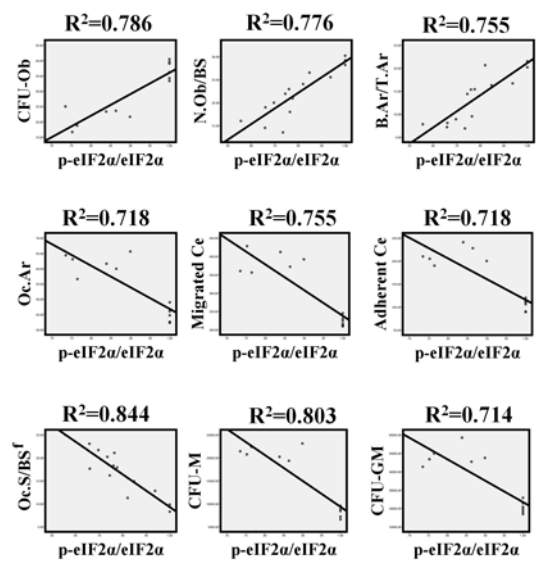

Figure 3 


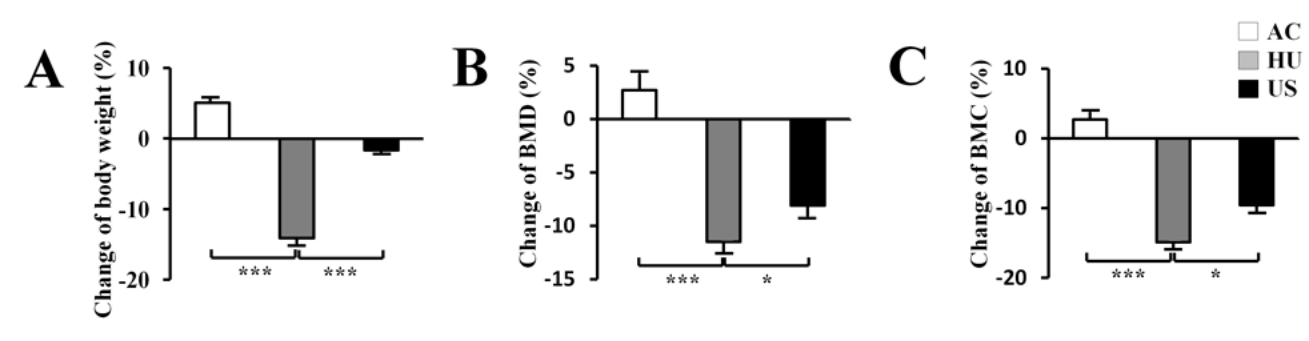

D
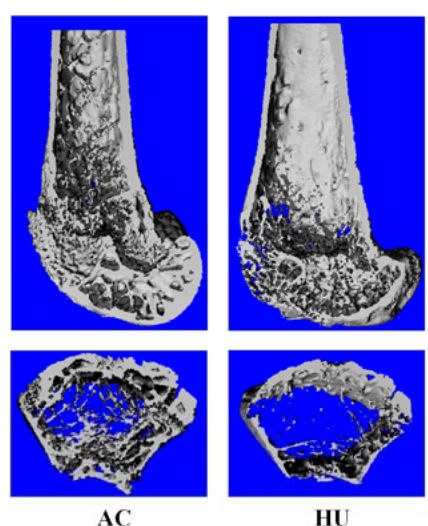

HU

G
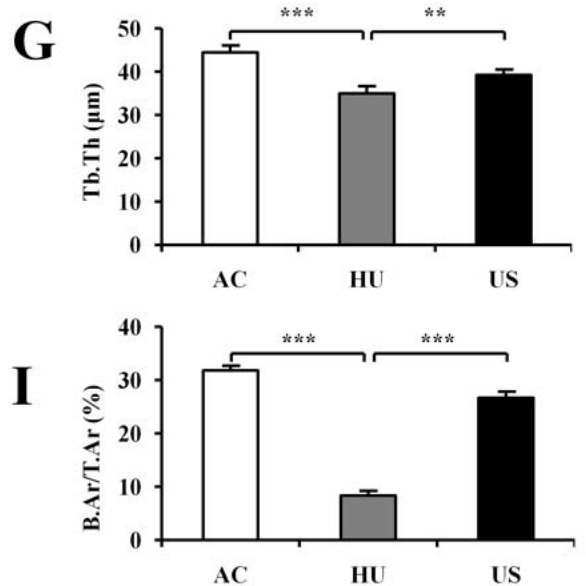
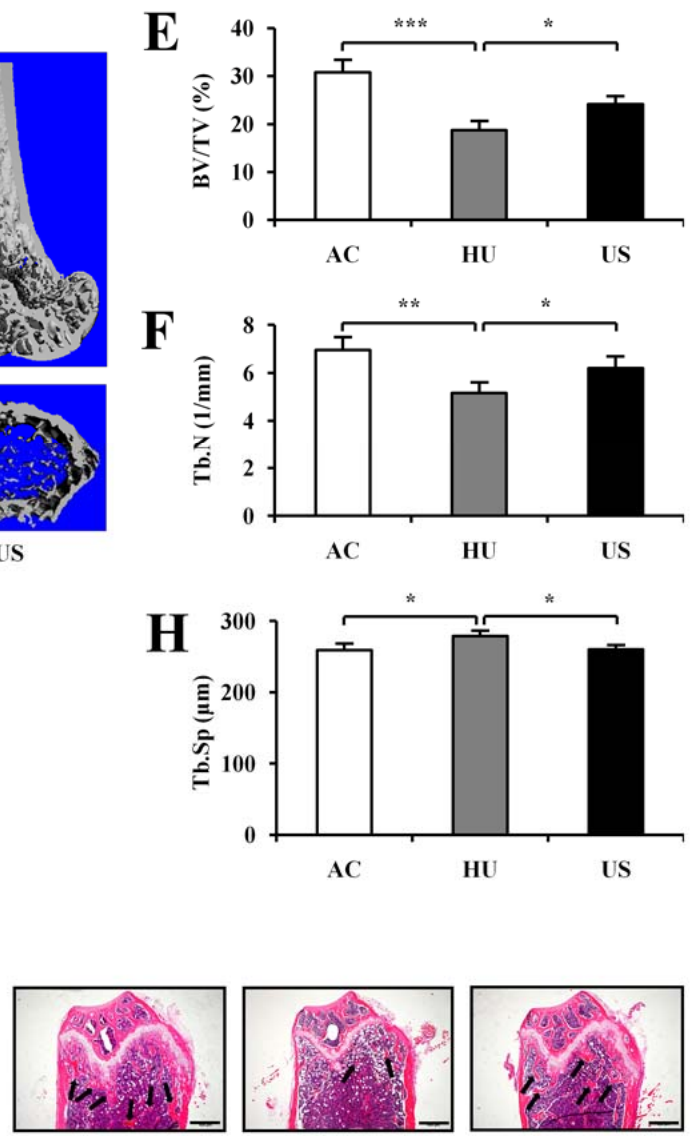

Figure 4 

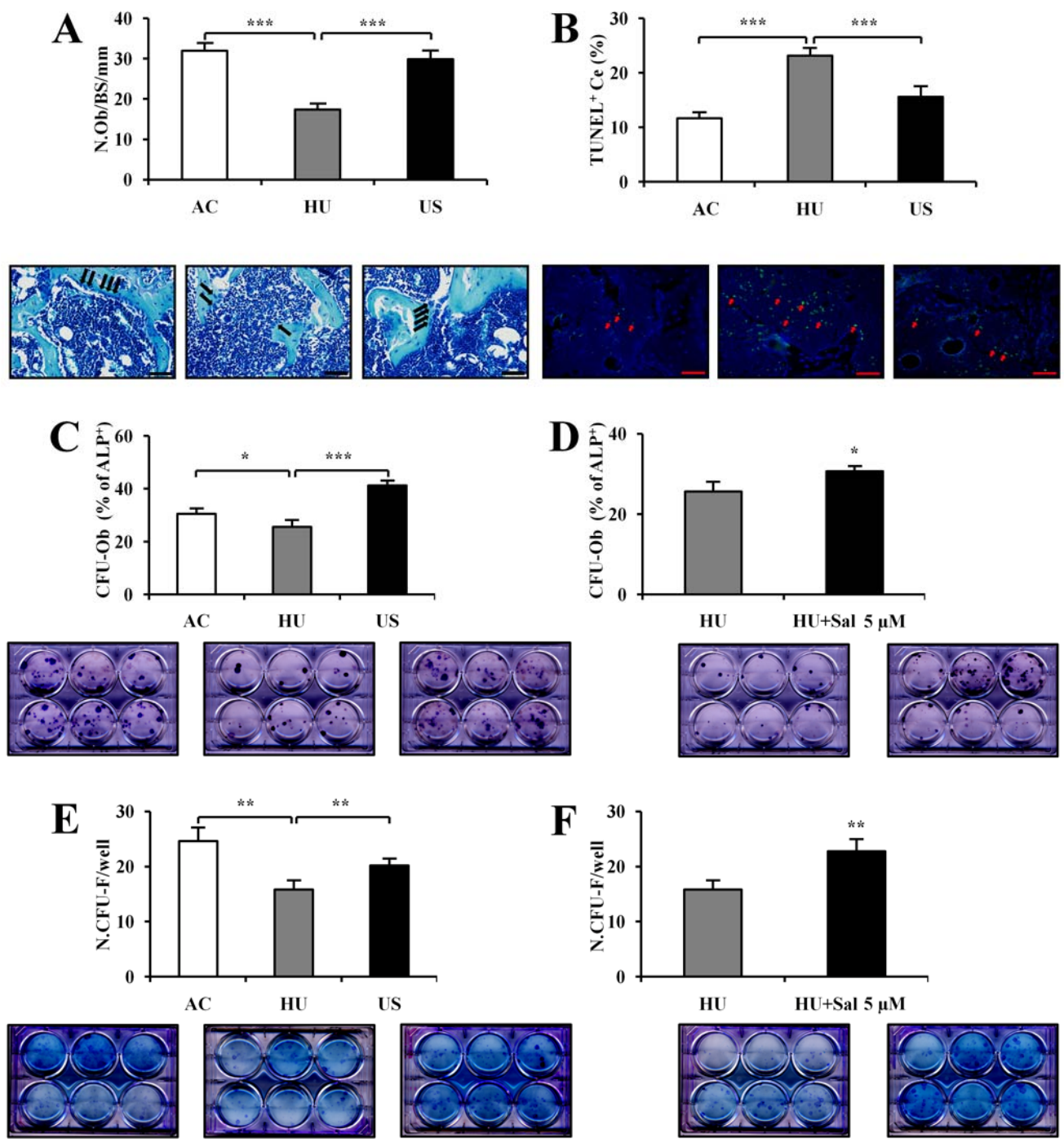

Figure 5 


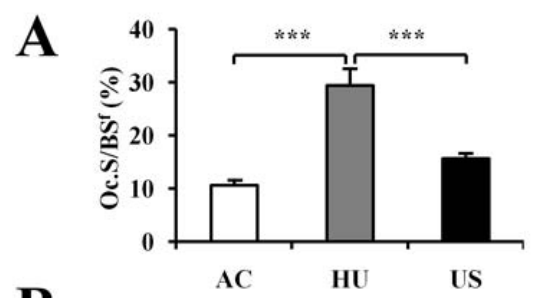

B
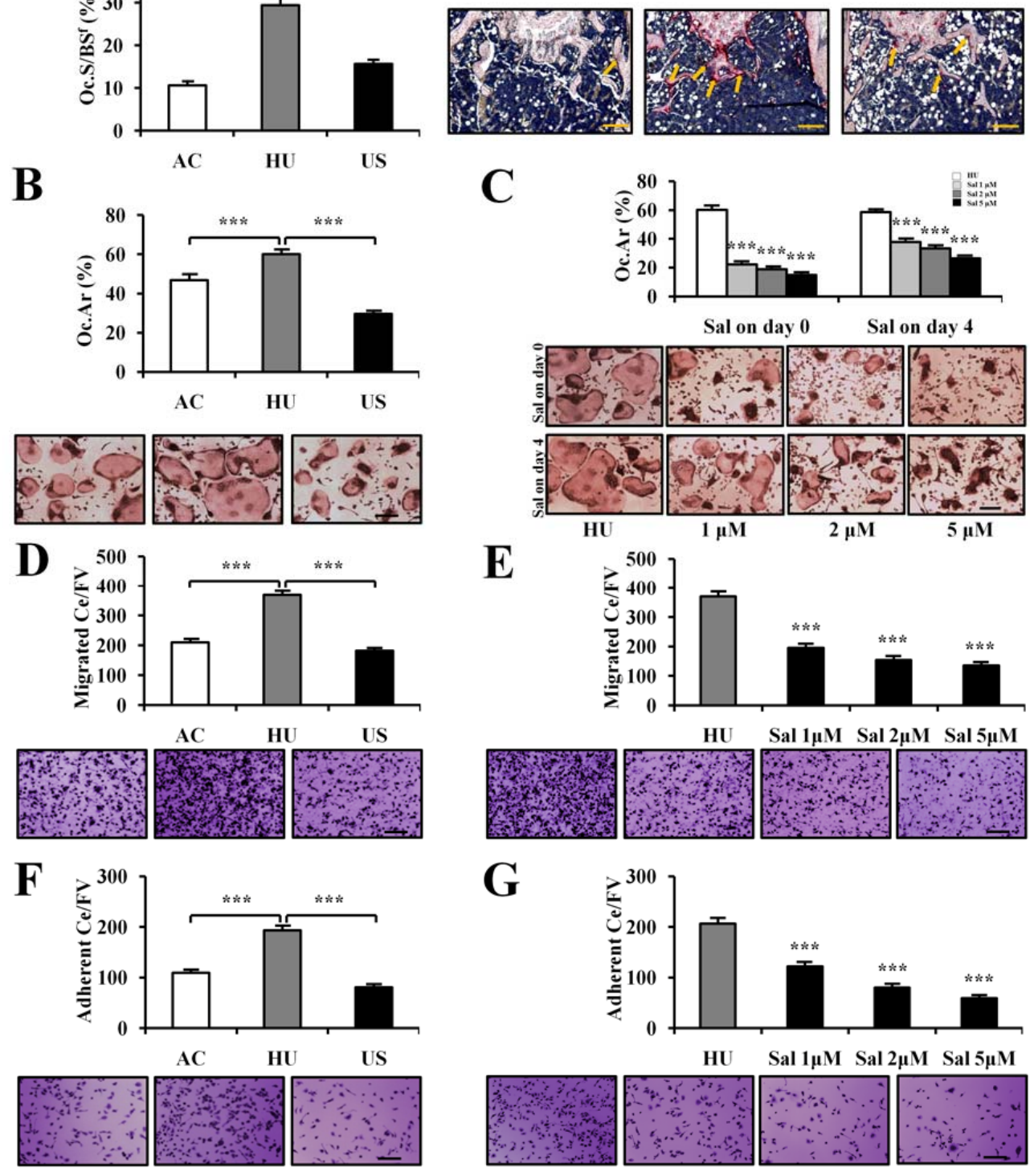

Figure 6 

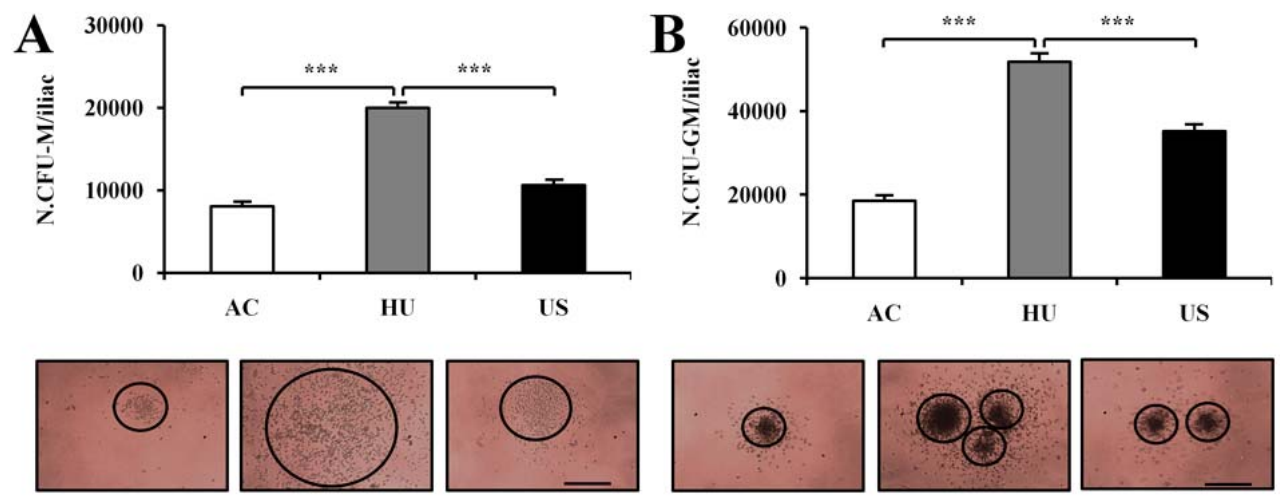

C
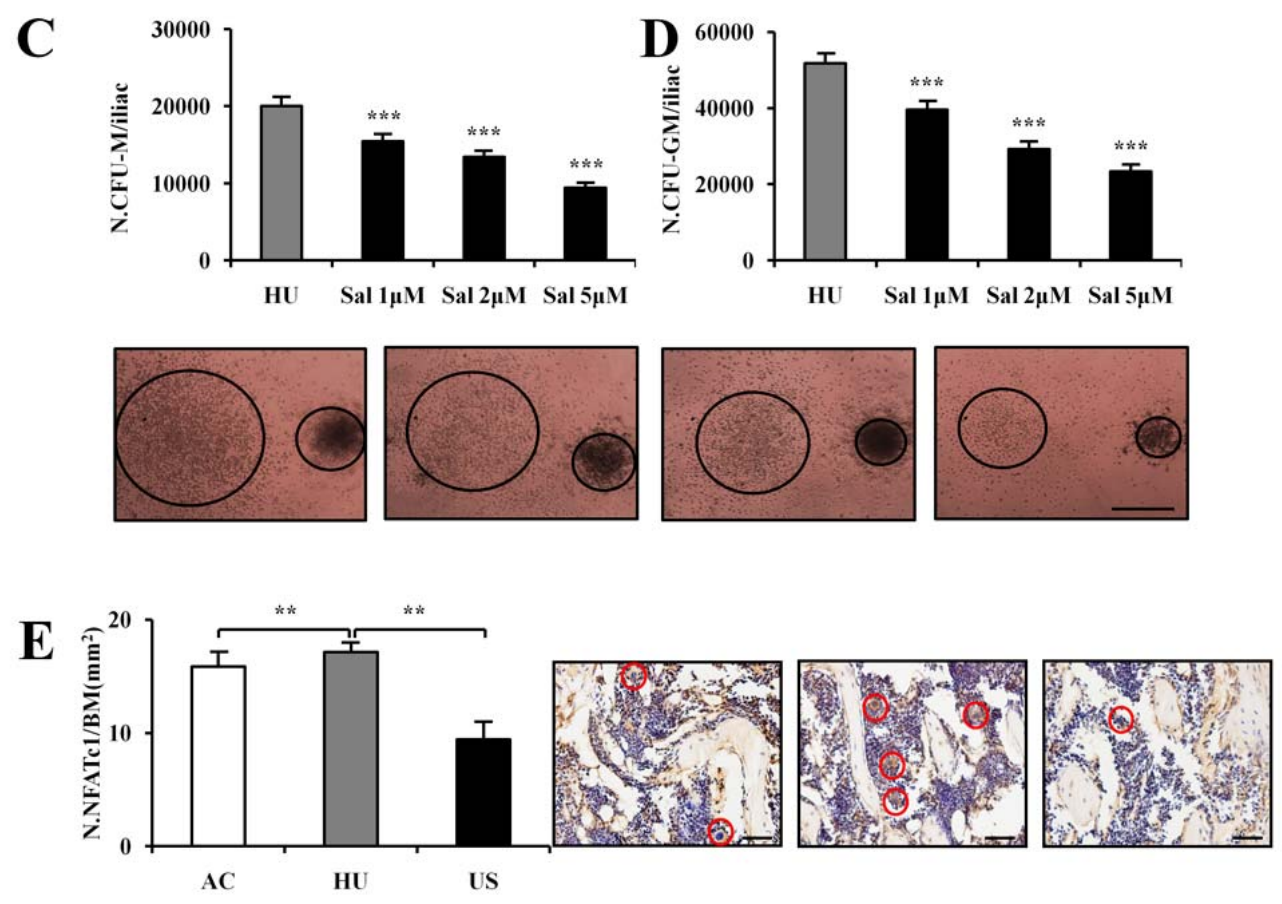

Figure 7 


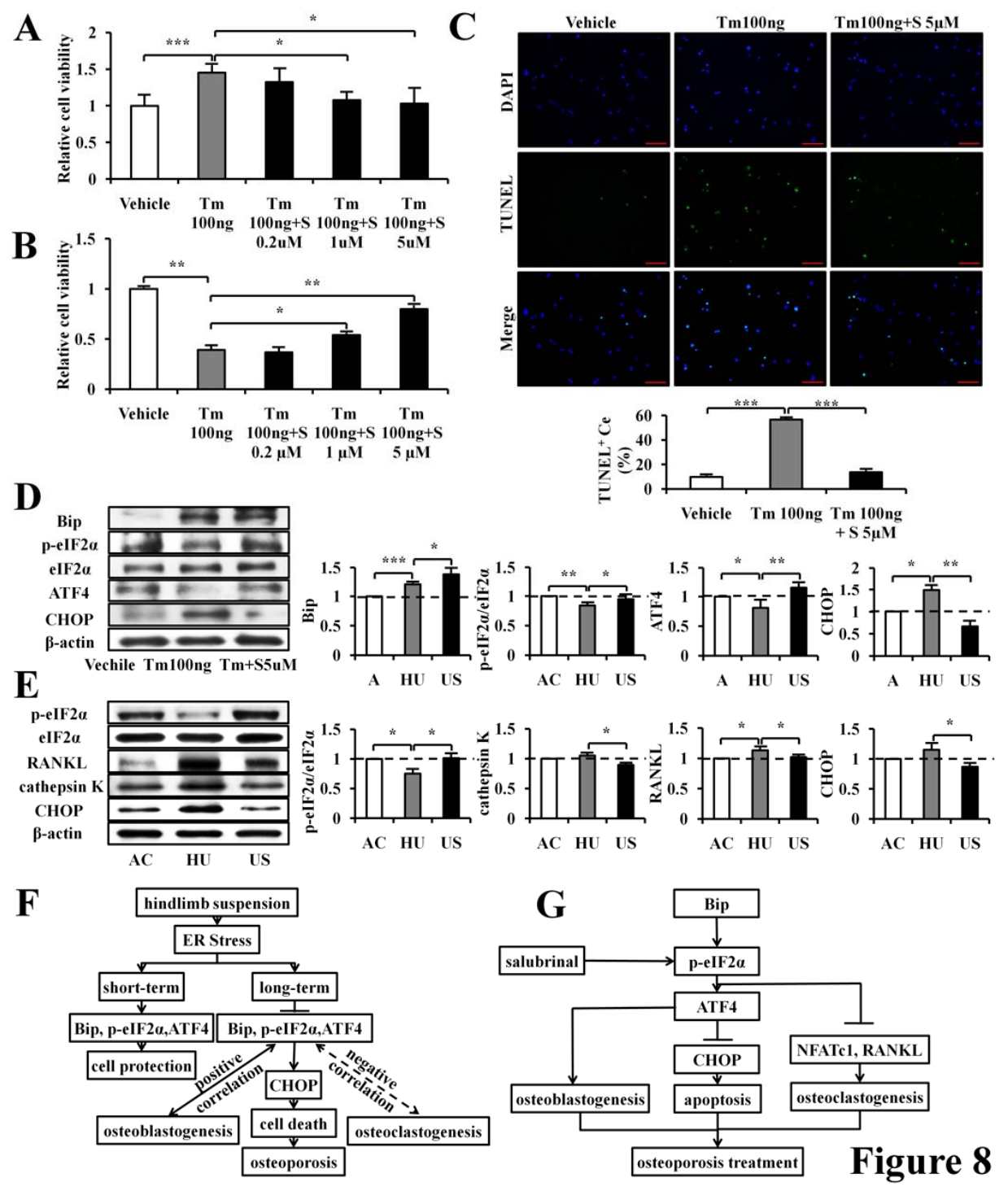

